Article

\title{
Intermodal Container Routing: Integrating Long-Haul Routing and Local Drayage Decisions
}

\author{
Hilde Heggen ${ }^{1,2, *(\mathbb{D})}$, Yves Molenbruch ${ }^{1,3} \mathbb{D}$, An Caris ${ }^{1}(\mathbb{D})$ and Kris Braekers ${ }^{1}(\mathbb{D})$ \\ 1 UHasselt, Research Group Logistics, Agoralaan, 3590 Diepenbeek, Belgium; \\ yves.molenbruch@uhasselt.be (Y.M.); an.caris@uhasselt.be (A.C.); kris.braekers@uhasselt.be (K.B.) \\ 2 Maastricht University, Department of Quantitative Economics, P.O. Box 616, \\ 6200 MD Maastricht, The Netherlands \\ 3 Research Foundation Flanders (FWO), Egmontstraat 5, 1000 Brussel, Belgium \\ * Correspondence: hilde.heggen@uhasselt.be; Tel.: +32-11-268786
}

Received: 28 February 2019; Accepted: 14 March 2019; Published: 18 March 2019

\begin{abstract}
Intermodal logistics service providers decide on the routing of demand through their service network. Long-haul routing decisions determine the selected departure and arrival terminals for containers and imply corresponding drayage tasks. Traditionally, given these long-haul routes and fixed drayage tasks, drayage operations are planned in a second phase by establishing truck routes to transport containers to and from terminals by truck. In this paper, operational decisions on local drayage routing in large-volume freight regions with multiple terminals on the one hand, and intermodal long-haul routing on the other hand are merged into an integrated intermodal routing problem. Different long-haul routing decisions imply different drayage tasks to be performed and thus impact total trucking costs. The approach aims at reducing the number of road kilometres and increases bundling opportunities by maximising the long-haul capacity utilisation. In this way, it contributes to the modal shift towards intermodal transport and a more sustainable transport system. As a weekly planning horizon is used, a maximum daily active time and a minimum overnight's rest are included for multi-day drayage routing. A large neighbourhood search heuristic is proposed to solve the integrated intermodal routing problem. This integrated planning approach provides decision support for routing customer orders throughout the intermodal network with the aim of minimising total transport costs and maximising capacity utilisation. Experiments show the added value of the integrated approach, which uses more information to make better-informed decisions and increase the capacity utilisation. The largest savings in trucking costs are obtained for clustered instances with demand characteristics closest to real-life cases. Finally, a real-life case study analyses the impact of tactical service network design decisions on the total operational costs.
\end{abstract}

Keywords: intermodal transport; integrated routing; intermodal routing; drayage; vehicle routing

\section{Introduction}

Forecasts indicate that European freight transport by road is expected to increase by $40 \%$ by 2030 and even by over $80 \%$ by 2050 compared to 2005. Despite the fact that road transport is less sustainable and environmentally friendly, it has the largest share in the modal split with $76.4 \%$, followed by $17.4 \%$ and $6.2 \%$ for rail and waterways respectively [1]. This expected continued growth in freight transport and its associated environmental impact present one of the major challenges in the transport sector [2,3]. Intermodal rail transport provides a promising opportunity to achieve a modal shift away from unimodal road transport and towards a more sustainable transport system. It combines the accessibility of road transport with economies of scale of rail transport. Furthermore, bundling load 
units on trains over long distances also relieves congested highways and reduces $\mathrm{CO}_{2}$-emissions and thus releases pressure on the environment [4].

Intermodal transport involves multiple actors and decision makers, resulting in a more complex planning environment compared to unimodal road transport. Synchromodal transport further increases this complexity as it requires informed and flexible planning [5]. The emergence of the Physical Internet [6], where containers are routed through an interconnected network of transport modes and routes, also creates new planning challenges. As the level of integration of different transport modes and decision levels influences the attractiveness of intermodal transport, adequate decision support systems for human planners at the operational decision level should be developed. Fast planning algorithms with real-life problem characteristics should be available to accommodate decisions in a complex intermodal setting.

Ambra et al. [7] emphasise this need for innovative integrated support systems with a focus on cost efficiency. For intermodal transportation to be advantageous, the integrated planning should be executed effectively and efficiently in order to minimise total transport costs and maximise service capacity utilisation, which in turn also results in decreasing costs of the transport system. Costs related to the execution of first- and last-mile drayage operations are an important aspect within this regard. At the same time, customer requirements should be respected. In addition, in order to get a competitive advantage compared to other intermodal operators, customers may be offered the possibility to book orders close to the required pick-up deadline for transport.

Intermodal logistics service providers must decide on the planning of drayage and main-haul transport. Tactical decisions are related to the selection of intermodal transport services and the allocation of existing resources [8]. The service network design determines the routes on which services are offered and the characteristics of each service [9]. Characteristics of regular long-haul services include the route, intermediary stops, frequency, vehicle and convoy type, capacity and speed. Most of these characteristics are unchanged for a given scheduling period [10]. Often, weekly planning cycles are fixed for at least six months. At the operational decision level, policies have to be determined for routing containers throughout the given intermodal network and services [9]. Intermodal routing or route selection is concerned with the selection of routes or itineraries for individual containers over a given intermodal network $[9,11]$, related to a planning horizon of one or several days. The problem also includes real-time itinerary replanning and asset management [12]. Next, during train load planning, containers are assigned to specific locations on intermodal trains while including detailed capacity considerations related to the wagon and slot length, and weight restrictions [13]. The fact that these problems and decision levels are interrelated is recently stressed by several authors (e.g., van Riessen et al. [14], Zhang and Pel [15]).

In current literature, decisions with respect to the long-haul transport and vehicle routing of trucks in a service region are usually considered independently. The distance or travel time between each customer location and the available terminals is taken into account when solving the intermodal long-haul routing problem. It results in a drayage task in each region. The truck cost is approximated by the direct distance between a customer and a terminal location. Clearly defined drayage operations are arranged in a second phase by combining pickup and delivery operations between customer locations and terminals into routes. In this sequential approach, the combination of pickup and delivery tasks in truck routes (i.e., the real total drayage cost) is ignored initially. However, the integration of vehicle routing problems into the intermodal terminal selection and container routing throughout the network may provide cost savings and a better utilisation of the available transport capacity.

In an integrated approach, the long-haul service is not fixed in advance, which results in multiple feasible drayage tasks per region, of which one per region has to be selected simultaneously with the decision on the long-haul service. In this paper, a model formulation and algorithm are proposed to compare the sequential and integrated approach, and quantify potential savings. The proposed integrated approach contributes to a more sustainable transport system by reducing the distances travelled using road transport. Moreover, by maximising the long-haul capacity utilisation, 
bundling opportunities can be fully deployed. A heterogeneous fleet of trucks and load units, multi-day truck scheduling and long-haul service capacity limits are considered. The focus is on rail transport for the long-haul, as most countries have access to an extensive rail network, whereas waterways are not always available. Figure 1 shows a possible truck cost reduction for the request indicated in black symbols by using an integrated approach. The selected long-haul service is indicated by the bold line between both regions. Whereas a sequential approach would select the long-haul service connecting both upper terminals, an integrated approach might select the lower service which may not have the lowest direct trucking distance, but results in the lowest total cost by combining different drayage tasks in a single truck route.

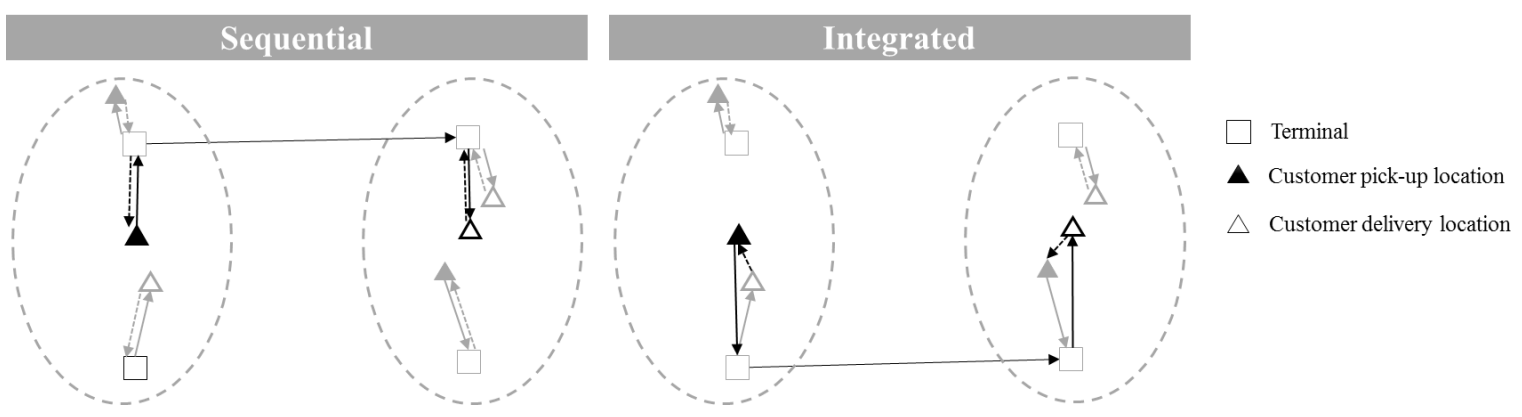

Figure 1. The sequential and the integrated approach-example.

In this paper, literature on both intermodal routing for the long-haul and intermodal vehicle routing problems and the contribution of this study are discussed (Section 2). The considered integrated planning problem is based on a real-life case study from the viewpoint of the transport planning department of an intermodal service operator with a network of long-haul services consisting of either own trains, purchased services which are managed by external parties, or a combination of both. Formulations for both subproblems in the sequential approach, as well as an integrated intermodal routing problem with real-life problem characteristics in which truck routing and long-haul routing decisions are made simultaneously, are proposed in Section 3. A large neighbourhood search algorithm is developed to solve the problem using both approaches, as discussed in Section 4. It includes a discussion on the multi-day scheduling procedure required to find feasible truck schedules. In Section 5, the sequential and integrated approach are compared by conducting computational experiments. Using the integrated approach, Section 6 presents a real-life case study and focuses on analysing the impact of tactical service network design decisions made by an intermodal service operator on the total operational costs. The main conclusions are summarised in Section 7.

\section{Related Literature}

The integrated intermodal routing problem relates to two problem settings in current literature which are usually solved independently: on the one hand routing containers (or load units) throughout the long-haul network, including service network design problems at the tactical level and intermodal routing at the operational level, and on the other hand vehicle routing problems in the context of drayage operations around terminals. Recent advances in literature on both types of problems are discussed in Sections 2.1 and 2.2 respectively. Section 2.3 presents the closest related research for integrating both problems. Section 2.4 concludes with the contribution of this paper compared to current research.

\subsection{Long-Haul Service Network Design and Intermodal Routing}

A literature survey on models and solution approaches for multimodal freight transport is presented by SteadieSeifi et al. [12]. Strategic, tactical and operational planning problems are discussed. The study shows that service network design and network flow planning problems are often solved using tabu search and neighbourhood search heuristics. 
Decision levels. Current research indicates that operational and tactical decisions are interrelated. Operational intermodal routing problems may include real-time planning and replanning $[11,14,16-18]$ and asset management (and repositioning) decisions [11,16,19-21]. They sometimes include fixed costs for selecting services, which relates to the tactical service network design. This can be explained by the fact that routes selected at the tactical decision level may no longer be feasible if disruptions occur at the operational level [16]. Tactical problems usually assume that delivery time windows can be violated, whereas at the operational phase, time windows are usually constraints. However, some operational problems allow late delivery at a penalty cost $[14,17,20,22]$. This shows that different decision levels are linked. Although some papers include more than one decision level, most research focuses on a single problem and decision level. This research combines two operational problems. The integrated problem is used to study the influence of changes in tactical decisions on operational transport costs.

Service characteristics and objectives. A mix of different types of transport services may be used, such as flexible or fixed, and own or externally managed services (e.g., Moccia et al. [23]). Flexible services can be activated ad hoc in accordance with the requirements, such as the decision to operate trucks or purchase slots on trains during peak periods, while fixed services usually provide a fixed capacity between a given departure and arrival location with a given schedule. Operators need to determine the capacity of own means and negotiate slot contracts with third parties based on estimated future demand. While services operated by other parties are generally purchased at a fixed cost per container, own services may be subject to economies of scale (e.g., Moccia et al. [23], Chang [24], Caramia and Guerriero [25]). For each service offered, current literature generally includes high-level capacity restrictions such as a maximum 'flow' or 'quantity'. Some papers include a total allowed maximum length in twenty foot equivalent units (TEU) or weight per service. From contacts with the transport sector, we learned that rail transport contracts with external parties include agreements which stipulate how much transport capacity is purchased. Slot agreements include detailed information on the number of slots, their (length) dimensions and the allowed weight for each selected slot. For own services, wagons are leased for a longer period based on expected transport requests. These aspects related to the determination of the mix of slot types should be determined at the medium term (i.e., tactical decision level). At the tactical level, van Riessen et al. [26] and Rudi et al. [27] consider a maximum weight and length, and number of containers and weight respectively. At the operational level, multiple types of capacity limits are included by van Riessen et al. [14], Bock [17], Moccia et al. [23] and Di Febbraro et al. [18].

Pre- and end-haulage costs are part of the total route costs, and are usually considered as direct links between shipper or receiver nodes and terminal nodes in the transport network. Combinations of pickup and delivery tasks during drayage operations (i.e., street turns) are not accounted for, but occur in practice.

\subsection{Pre- and End-Haulage Transport}

Intermodal local drayage operations are concerned with the pickup and delivery of full-truckload inbound and outbound containers in the service region of an intermodal terminal within time windows at terminals and customer locations [28]. The aim is to find efficient truck routes between intermodal terminals and customer locations, where trucks typically have a capacity of a single container. Total transport distances and costs can be reduced by allowing direct transport between customer locations (i.e., street turns), which reduce empty transport distances. For a state of the art on general vehicle routing problems, the reader is referred to Toth and Vigo [29] and Braekers et al. [30].

The vehicle fleet is often considered homogeneous; only two papers discuss a heterogeneous fleet [31,32]. Multiple vehicle depots can be included [33-38]. Moreover, to cope with imbalances between demand and supply of containers at different locations, the allocation of empty containers can be modelled [32-40]. Time windows at intermodal terminals reflect either opening hours of terminals 
or ultimate arrival times for a long-haul service selected in advance. In the latter case, the long-haul service network and routing characteristics are implicitly included and assumed to be given.

Although several papers include multiple terminals [32,34-36,38-40], it is generally assumed that the pickup location (i.e., arrival terminal) of inbound full containers and delivery location (i.e., departure terminal) of outbound full containers are known and fixed. This implies that long-haul routing decisions are assumed to be given.

\subsection{Research Opportunities}

Integrating the decision on which pickup tasks and delivery tasks to combine in truck routes when making intermodal routing decisions may lead to reduced trucking costs and possibly different long-haul itineraries for individual containers. The integration of both interdependent problems, the determination of long-haul routes and drayage routes, into an integrated intermodal container routing problem may result in important cost savings. However, compared to our model, research efforts which simultaneously consider both decisions are still limited.

In the literature on dial-a-ride problems, Posada et al. [41] integrate transfers to and from timetabled public transport into the classical dial-a-ride problem. Decisions on which transfer terminals to use (and thus which public transport line) are made simultaneously with vehicle routing decisions. The closest related research on freight transport consists of several recent research efforts. Medina et al. [42] synchronise the service network design and vehicle routing problem by assigning each customer location to a single terminal. Dragomir et al. [43] formulate a less-than-truckload (LTL) pickup and delivery problem with interregional lanes for long-haul vehicles between multiple regions, using multiple depots transport modes. No solution method is provided. Soriano et al. [44] solve a two-region LTL multi-depot pickup and delivery problem. However, long-haul schedules and capacity limits for the main-haul, multi-day truck scheduling and heterogeneous vehicles are not considered. Wolfinger et al. [45] also consider transport over longer distances using multiple transport modes. They include a limited daily active time, but no minimum overnight's rest. Further, as schedules or capacity restrictions for the long-haul are ignored, trade-offs between drayage costs and long-haul routing cannot be examined.

Moccia et al. [23] focus on the decision of which mix of fixed and flexible (requested ad hoc) services should be activated from other operators, given the fact that certain train services are purchased with train weight and length limits. Their problem is clearly related. However, truck routing in a service region and detailed capacity considerations are not included. With respect to intermodal drayage, only one paper [32] includes a flexible terminal assignment cost in a vehicle routing problem within the service region of an intermodal terminal. However, no long-haul service characteristics are incorporated. To the best of our knowledge, no paper considers both real-life rail service and drayage characteristics and handles intermodal routing and drayage in an integrated way.

With respect to capacity levels, clear differences exist between literature on intermodal routing and train load planning. Current research on train load planning includes detailed capacity requirements (i.e., size and weight) when containers are already assigned to specific rail routes. On the other hand, problems aimed at routing containers throughout a service network generally consider a very high-level view on capacity (e.g., several containers). Furthermore, in practice, at the tactical decision level, the intermodal operator determines slots to be purchased on externally managed trains and wagon lease agreements of own services. This relates to the physical dimensions of transport requests at the operational level (e.g., $30 \mathrm{ft}, 45 \mathrm{ft}$ ). When demand for transport requests is high, capacity limits of slots of each dimension on external services or the total available length or weight for own services may influence the selected long-haul route for individual requests (e.g., if not enough capacity is available of a specific dimension at one service, another should be selected). Within this regard, a heterogeneous fleet of trucks is also employed for containers of varying dimensions. These more detailed capacity requirements related to the dimensions of slots, wagons and trucks must be integrated into container routing decisions. 


\subsection{Contributions}

To the best of our knowledge, a new, integrated intermodal routing problem is proposed and compared with a sequential approach in which first intermodal routing decisions and next decisions on drayage operations are made. An integrated planning approach simultaneously decides on the routing of containers throughout the network and local truck routing with a heterogeneous truck fleet in each service region. By considering drayage and long-haul costs simultaneously, total transport costs may be reduced. In this integrated approach, the customer pickup location for outbound containers and the customer delivery location of inbound containers within each service region are known and time windows at customer locations are fixed. A container can be transported via any available long-haul connection, implying that the departure and arrival terminal are not fixed.

Capacity considerations are added which are generally not dealt with in intermodal routing, such as length and weight limits. This is in line with operational problems such as the train load planning problem, which does include a very detailed level of capacity limits. Service contracts with other transport operators include detailed capacity information, such as several slots per length type and weight category. Moreover, not all containers can be carried by any truck, depending on the dimensions of containers and trucks. This may result in separate routes for different types of containers and trucks. Different container types related to the physical dimensions are assigned to the appropriate slot dimensions for long-haul rail services and with the routing of a heterogeneous fleet of trucks for pre- and end-haulage operations. While trucks start and end their route at a depot located at a terminal, they can be out for multiple days before returning to the depot. An overnight's minimum resting period is enforced at any location after a maximum daily active time. These operational considerations influence the routing decisions and should therefore be included.

The integrated planning approach, with capacity considerations for trucking and rail planning, is aimed at maximising the overall long-haul network capacity utilisation and minimising total transport costs. In the next sections, operational capacity requirements are included in decisions on both routes of containers throughout a service network and intermodal drayage routes for pickups and deliveries. The goal is to analyse potential savings by using an integrated approach, and to provide insights in how to best use the current network for intermodal activities.

\section{Problem Formulation}

An intermodal service operator considers several full-container requests which should be transported from their customer origin location to their customer destination location through an intermodal network with two large service regions. Within each service region, local pre- and end-haul operations should be performed. Between both service regions, long-haul transport is available. No unimodal direct trucking between customer origins and destinations is allowed, which is acceptable if the distance between the two service regions is relatively high. The aim is to minimise the total transport cost, which consists of both rail transport and trucking costs.

Each request has a known weight, length (or container type), and time window within which it can be transported, and must be routed throughout the intermodal network from its customer origin location to its customer destination location. Time windows consist of an earliest pickup time at the customer origin location (i.e., release time of a request) and a latest arrival at the customer delivery location (i.e., due time of a request).

The long-haul network consists of several terminals in each service region. Links representing available services connect the terminals in periodical, usually weekly, planning cycles. Each rail service is characterised by a capacity limit, a departure terminal and an arrival terminal, a cutoff time before which containers using that service must arrive at the departure terminal and a time at which the containers are released at the arrival terminal, after which the containers are available for pickup by trucks for last-mile drayage. Multiple services with the same departure and arrival terminal may exist with different departure days and times. It is assumed that the available scheduled services are known and a long-haul service has to be selected for each load unit. The rail haul cost for transporting a 
request using a service depends on the service and the container type of each request. An example of a network is visualised in Figure 2.

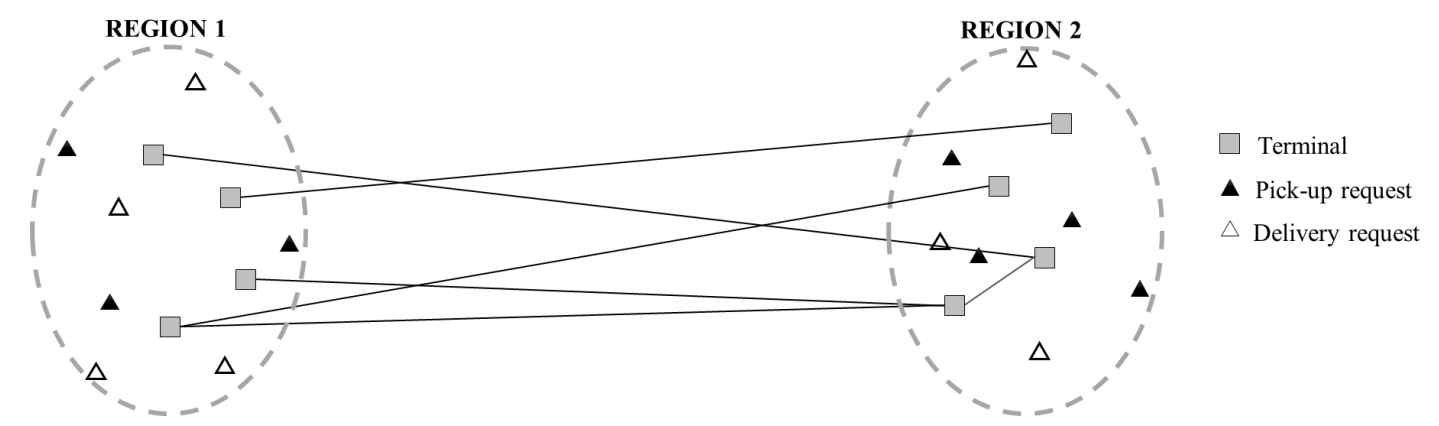

Figure 2. Two-region intermodal network.

Within each service region, trucks perform pickup and delivery operations. Each request requires two truck drayage tasks, one pickup task from its customer origin location to the selected terminal in the service region, and one delivery task from a terminal in another region to its customer destination location. Moreover, it is assumed that empty container repositioning between terminals is determined afterwards in accordance with the demand for empty containers. Truck depots are located at some of the terminals. Containers never stay at a customer location, as trucks wait for loading or unloading. A limited, heterogeneous fleet of trucks, with a capacity of a single container, is available, with as many truck types as container types. Separate truck routes for each type of container must be established. Truck costs are assumed to be proportional to the travelled distance. Trucks depart at the start of the planning horizon at a depot and return before the end of the planning horizon, consisting of multiple consecutive days, to the same depot. After performing pickup an delivery operations during a maximum daily active time, trucks should spend the night at any node in the network for a minimum overnight's rest time.

Flows in both directions between the service regions are considered to route containers in two ways between the regions, which implies that the truck routing in each of the regions includes both pickup tasks of containers at customers to deliver at terminals and delivery tasks of containers picked up at terminals to customer locations.

This results in the following notation.

Sets and indices

$$
\begin{aligned}
K= & \{1, \ldots,|K|\}=\text { set of vehicles, index } k \\
D= & \{1, \ldots,|D|\}=\text { number of days in the planning horizon, index } d \\
R= & \{1, \ldots,|R|\}=\text { set of containers (requests), index } r \\
N^{P}= & \{1, \ldots,|R|\}=\text { set of customer pickup locations, index } g, h \text {, one per request } \\
N^{D}= & \{|R|+1, \ldots, 2|R|\}=\text { set of customer delivery locations, index } g, h, \\
& \text { one per request, with delivery node }|R|+g \text { related to pickup node } g \in N^{P} \\
N^{T}= & \{2|R|+1, \ldots, 2|R|+m\}=\text { set of } m \text { terminal nodes, index } g, h \\
N= & \{1, \ldots, 2|R|+m\}=\text { node set with terminals, pickup and delivery nodes } \\
= & N^{P} \cup N^{D} \cup N^{T} \\
V= & \{1, \ldots,|V|\}=\text { possible physical dimensions for containers/trucks/slots, index } v \\
S_{\text {own }}= & \text { set of own long-haul services } \\
S_{\text {ext }}= & \text { set of externally owned long-haul services } \\
S= & \text { set of long-haul services with index } s \text { between terminals, } \\
& \text { where } S=S_{\text {own }} \cup S_{\text {ext }}
\end{aligned}
$$




\section{Parameters}

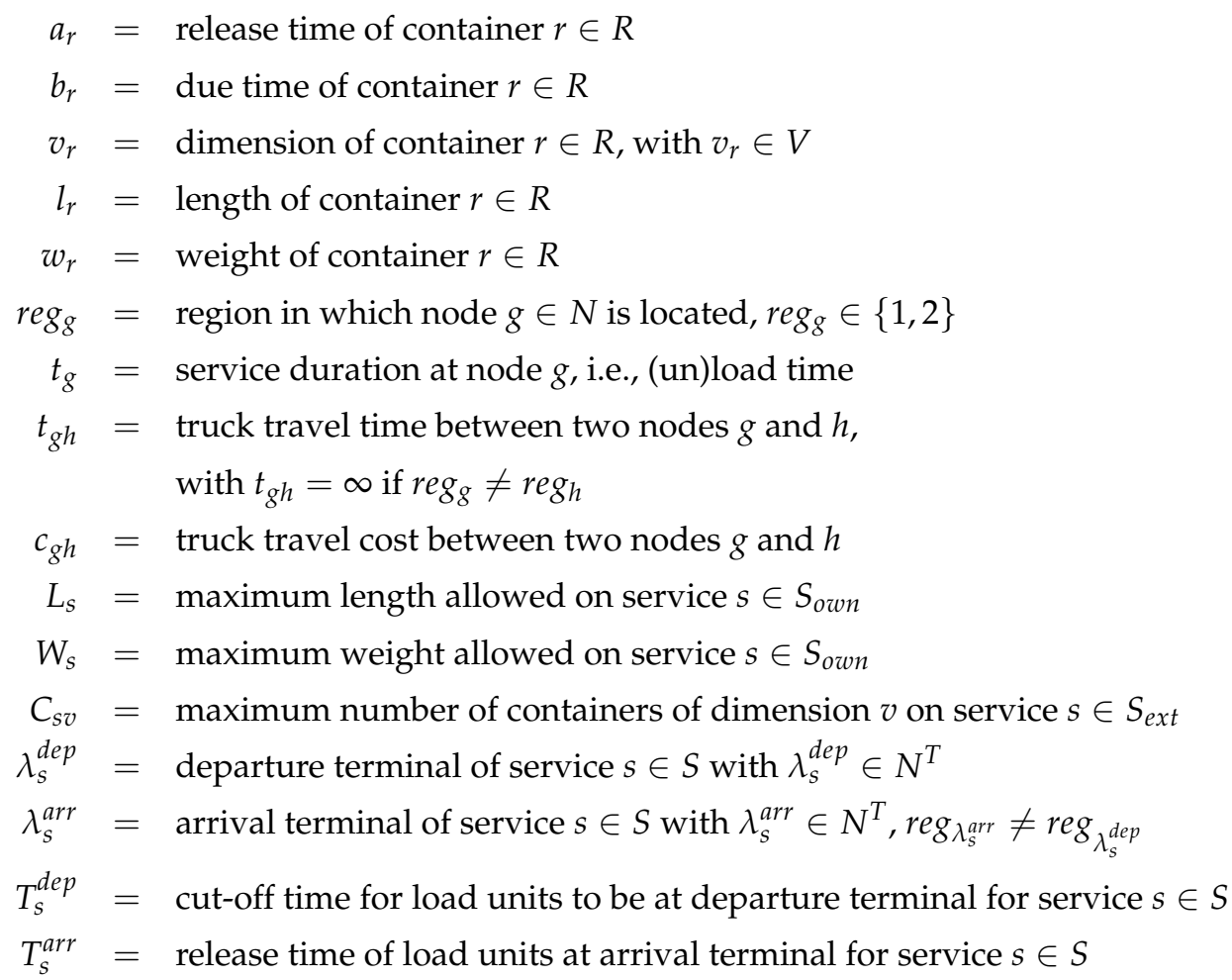

The problem will be solved in the following two ways in order to compare an integrated approach with a sequential approach. The sequential method first assigns each request to a long-haul service. A request can only be assigned to a service if the departure terminal can be reached in time with a direct truck route from the customer pickup location to the departure terminal, and the customer delivery location can be reached in time with a direct truck route from the arrival terminal to the customer delivery location. Furthermore, capacities of the services are accounted for. The objective is to find the least-cost assignment, where trucking costs are approximated using the direct distances between customer locations and the selected terminals. Consequently, a pickup task-from a customer pickup location to a known departure terminal—and a delivery task-from a known arrival terminal to a customer delivery location-are defined for each request. In a second phase, for each region these drayage tasks are combined into truck routes which minimise the total trucking cost. The integrated approach considers both the service selection and the truck routing simultaneously. This implies that the entire truck route costs are considered when assigning requests to services, instead of just the transport costs incurred by travel between a customer location and a terminal.

Both subproblems of the sequential approach are discussed in Section 3.1. The section presents a mathematical formulation for the intermodal long-haul routing problem for routing containers through a given intermodal network of long-haul services, as well as the vehicle routing problem used to minimise trucking costs for local pickups and deliveries of full containers. Finally, both problems are considered simultaneously in Section 3.2 in order to minimise the total operational cost of the intermodal truck-rail transport network using an integrated approach.

\subsection{The Sequential Approach}

\subsubsection{The Intermodal Long-Haul Routing Problem}

Given the available periodic service schedules and characteristics, the aim of the intermodal routing problem is to determine the movement of requests throughout the given network in order to minimise total transport cost, consisting of the pre-haul trucking cost from a customer pickup location 
and the departure terminal, long-haul rail costs, and end-haul trucking from the arrival terminal to the customer delivery location. The problem is formulated as follows:

Parameters

$$
\begin{aligned}
c_{r s}^{\text {pre }}= & \text { trucking cost between customer origin location of } r \text { and departure } \\
& \text { terminal of service } s \\
c_{r s}^{\text {end }}= & \text { trucking cost between arrival terminal of service } s \text { and customer } \\
& \text { destination location of } r \\
c_{r s}^{r a i l}= & \text { rail cost for container } r \text { on service } s \\
t_{r s}^{p r e}= & \text { trucking time between customer origin location of } r \text { and departure } \\
& \text { terminal of service } s \\
t_{r s}^{\text {end }}= & \text { trucking time between arrival terminal of service } s \text { and customer } \\
& \text { destination location of } r
\end{aligned}
$$

\section{Decision variables}

$$
X_{r s}=\left\{\begin{array}{l}
1, \text { if container } r \text { uses rail service } s \\
0, \text { else }
\end{array}\right.
$$

For a single planning period, which consists of a one-week planning cycle, the following problem can be formulated.

\section{Problem $P_{1}$ :}

$$
\min \sum_{r \in R} \sum_{s \in S}\left(c_{r s}^{p r e}+c_{r s}^{r a i l}+c_{r s}^{\text {end }}\right) \cdot X_{r s}
$$

subject to

$$
\begin{array}{lr}
\sum_{s \in S} X_{r s}=1 & \forall r \in R \\
\sum_{r \in R \mid v_{r}=v} X_{r s} \leq C_{s v} & \forall s \in S_{\text {ext }} ; v \in V \\
\sum_{r \in R} l_{r} \cdot X_{r s} \leq L_{s} & \forall s \in S_{\text {own }} \\
\sum_{r \in R} w_{r} \cdot X_{r s} \leq W_{s} & \forall s \in S_{\text {own }} \\
a_{r}+t_{r}+t_{r s}^{p r e}+t_{\lambda_{s}^{\text {dep }}} \leq T_{s}^{\text {dep }}+\left(1-X_{r s}\right) \cdot M_{r s}^{1} & \forall r \in R ; s \in S \\
T_{s}^{\text {arr }}+t_{\lambda_{s} \text { arr }}+t_{r s}^{\text {end }}+t_{r+R} \leq b_{r}+\left(1-X_{r s}\right) \cdot M_{r s}^{2} & \forall r \in R ; s \in S \\
X_{r s} \in\{0,1\} & \forall r \in R ; s \in S
\end{array}
$$

The objective is to minimise total transport costs (1). Exactly one service is to be selected for each load unit, as indicated by constraints (2). The total capacity between two rail nodes is limited by constraints (3)-(5). The heterogeneous slots purchased on external long-haul services are linked with containers of the same dimensions by constraints (3). For own long-haul services, constraints (4) and (5) impose a maximum length and weight respectively. It is assumed that a feasible assignment of containers to the specific locations on wagons can be found using a detailed train load planning algorithm as for example proposed in Heggen et al. [13]. Furthermore, each container $r$ can only be assigned to a specific rail service if its release time $a_{r}$ at the customer increased with the time needed to arrive at the considered terminal allows the container to arrive before the service departure (6). Each container can only leave a terminal after release 
at the arrival terminal of the selected service, and must arrive at its customer delivery location before its due date $b_{r}$ (7). For these time constraints, $M_{r s}^{1}=\max \left\{0 ; a_{r}+t_{r}+t_{r s}^{p r e}+t_{\lambda_{s}}{ }_{\text {dep }}-T_{s}^{d e p}\right\}$, and $M_{r s}^{2}=\max \left\{0 ; T_{s}^{a r r}+t_{\lambda_{s}^{a r r}}+t_{r s}^{\text {end }}+t_{r+R}-b_{r}\right\}$ respectively. Finally, the decision variables $X_{r s}$ are binary (8).

\subsubsection{Vehicle Routing Problem in Each Region}

Within the scope of this problem, it is assumed that the terminal at which each container should be picked up or delivered, is known and fixed because the long-haul problem has been solved first. Total truck route costs in each of the service regions should be minimised, given pickup and delivery locations of containers. Additionally, separate truck routes are established for each vehicle type and related container dimensions $v$. This results in $2 \cdot|V|$ routing problems, one for each vehicle type in each region. The proposed formulation can solve these problems all at once.

Each container (or request) requires two drayage tasks. Each drayage task has a pickup node $g$ and delivery node $h$, of which one is always a customer pickup or delivery location and the other a terminal location to drop off or pick up a container. Depots $i \in N^{D e p}$ at which trucks must start and end their routes are located at at least one terminal location per region. The resulting problem is a full-truckload pickup and delivery problem with time windows.

The problem can be formulated as an asymmetric multiple vehicle travelling salesman problem with time windows (am-TSP-TW) [40], in which a pickup node $g$ and its corresponding delivery node $h$ are merged into a single node $i$ and the distance between two nodes $i$ differs depending on the travel direction. Using the notation of Braekers et al. [40], each node $i \in O$ can be:

- $\quad$ a depot node $\left(i \in O^{D e p}\right), O^{\text {Dep }}$ identical to $N^{\text {Dep }}$

- a pickup task $\left(i \in O_{r}^{\text {pre }}\right)$, which consists of a customer pickup location $\left(g \in N^{P}\right)$ and a terminal $\left(h \in N^{T}\right)$, both located in the same service region.

- an delivery task $\left(i \in O_{r}^{\text {end }}\right)$, which consists of a terminal $\left(g \in N^{T}\right)$ and a customer delivery location $\left(h \in N^{D}\right)$.

This implies the following characteristics of each node $i$ :

- $\quad$ a service time $t_{i}=t_{g}+t_{g h}+t_{h}$, with $t_{i}=0 \forall i \in O^{D e p}$.

- $\quad$ a cost of visiting a node $c_{i}=c_{g h}$, with $c_{i}=0 \forall i \in O^{D e p}$.

- a node type $v_{i}$, related to the request dimensions. If the node is a pickup task $\left(g \in N^{P}\right.$, and consequently $\left.h \in N^{T}\right), v_{i}=v_{g}$, while $v_{i}=v_{h}$ if it is a delivery task $\left(h \in N^{D}\right.$, and consequently $\left.g \in N^{T}\right)$.

- $\quad$ a time window $\left[a_{i}, b_{i}\right]$ within which service at node $i$ should start. For pickup tasks $\left(g \in N^{P}\right)$ : $a_{i}=a_{g}, b_{i}=T_{s}^{d e p}-t_{i}$. For delivery tasks $\left(h \in N^{D}\right): a_{i}=T_{s}^{a r r}, b_{i}=b_{h}-t_{i}$.

- $\quad$ a rail service $\sigma_{i} \in S$, related to node $i$.

As a rail service $\sigma_{i}$ is selected for each request in a previous phase, one pickup and one delivery node related to each request are known. Routes have to be established in which different nodes are linked, and each truck must start and end its route at a depot. To be able to limit the total maximum duration of a truck route, each depot node $i \in N^{D e p}$ is duplicated two times the maximum number of vehicles of the considered type available at the depot. This results in $d$ depot nodes, with $d=2 *$ Vehicles $*$ Depots, as each route $k$ must start at start depot $i=O_{k}^{\text {Dep }}$, and end at its related end depot $j=O_{k}^{\text {Depe }}=i+d / 2$. Physical dimensions differ per truck, and the number of trucks of each dimension is limited in each region.

It would not be realistic to assume that drivers can continue their routes infinitely until the end of the planning horizon. To guarantee a limited daily active route duration and impose a minimum overnight rest, variable $m_{k i}^{d}$ is added, which is a binary variable that indicates whether truck $k$ spends the night following day $d$ at node $i$. A binary variable $\alpha_{i}^{d}$ indicates whether the service in node $i$ is 
performed at any day or time before night $d\left(\alpha_{i}^{d}=1\right)$ or after night $d\left(\alpha_{i}^{d}=0\right)$. In the formulation, the value of the variable $\alpha_{i}^{d}$ is only relevant if $\sum_{k \in K} m_{k i}^{d}=1$. During the execution of a single drayage task (represented by the service of node $i$ ), the truck is loaded, and trucks are not allowed to take an overnight rest. As the last night of the planning horizon constitutes the end of the planning horizon, no overnight stay is included for the night after the last day of the planning horizon.

The compatibility between two nodes in a given route (i.e., for a single truck) $c p_{i j k}$ indicates whether the truck type available for a route $k$ is compatible with two nodes $i$ and $j$. Depot nodes are characterised by the dimensions $v$ of the truck linked to that route. Therefore, node $i$ and $j$ are compatible in the route of truck $k\left(c p_{i j k}=1\right)$ if their dimensions are identical $\left(v_{i}=v_{j}=v_{k}\right)$, i.e., two identical types of requests with the same dimensions and a truck with the same dimensions, and if they are located in the same region $\left(i \neq j, r e g_{i}=r e g_{j}, r e g_{i}=r e g_{k}\right)$. Finally, $M$ is a sufficiently large number.

The following additional notation is used.

Sets:

Parameters:

$$
\begin{aligned}
O^{\text {Deps }} & =\text { set of start depots, index } i, j \\
O_{k}^{\text {Dep }} & =\text { start depot of vehicle } k \text {, index } i, j \\
O^{\text {Depe }} & =\text { set of end depots, index } i, j \\
O_{k}^{\text {Depe }} & =\text { end depot of vehicle } k \text {, index } i, j \\
O^{\text {Dep }} & =\text { set of depots, index } i, j \\
& =\bigcup_{k} O_{k}^{\text {Dep }} \cup \cup \bigcup_{k} O_{k}^{\text {Depe }}, \text { with one duplicate depot per route/vehicle } k \\
O_{r}^{\text {pre }} & =\text { pickup task for request } r, \text { indices } i, j \\
O_{r}^{\text {end }} & =\text { delivery task for request } r, \text { indices } i, j \\
O & =O^{\text {Dep }} \cup \bigcup_{r} O_{r}^{\text {pre }} \cup \bigcup_{r} O_{r}^{\text {end }}, \text { set of nodes, indices } i, j
\end{aligned}
$$

$Q_{d}^{a}=$ earliest time at which trucks can depart after an overnight's rest at the end of day $d$, with $1 \leq d<|D|$

$Q_{d}^{b}=$ latest time at which trucks finish their active time before an overnight's rest at the end of day $d$, with $1 \leq d<|D|$

$T_{\max }=$ maximum daily duration of a single truck route

$T_{\text {min }}=$ minimum night's rest between routes on two consecutive days

$c p_{i j k}=\left\{\begin{array}{l}1, \text { if nodes } i \in O, j \in O \text { in route } k \text { are compatible } \\ 0, \text { else }\end{array}\right.$

$c_{i j}^{T}=$ trucking cost of travelling from the end of node $i$ to the start of node $j$

$c_{i}=$ cost of serving node $i$

$t_{i}=$ service time at node $i$

$\sigma_{i}=$ rail service related to pickup or delivery node $i \in O \backslash O^{D e p}$

$t_{i j}=$ trucking time between nodes $i$ and $j$

\section{Decision variables}

$A_{i}=$ arrival time at node $i \in O$

$B_{i}=$ begin time of service at node $i \in O$ 


$$
\begin{aligned}
& D_{i}=\text { time at which a vehicle leaves the destination location of task } i \in O \\
& B T_{k}^{d}=\text { start time of route } k \text { on day } d+1 \text {, with } 1 \leq d<|D| \\
& E T_{k}^{d}=\text { end time of route } k \text { on day } d \text {, with } 1 \leq d<|D| \\
& m_{k i}^{d}=\left\{\begin{array}{l}
1, \text { if vehicle } k \text { spends the night following day } d \text { at node } i \\
0, \text { else }
\end{array}\right. \\
& \alpha_{i}^{d}=\left\{\begin{array}{l}
1, \text { if service of } i \text { is performed before the night following day } d \\
0, \text { else }
\end{array}\right. \\
& Y_{i j k}=\left\{\begin{array}{l}
1, \text { if } \operatorname{arc}(i, j) \text { is used in route } k, \text { with } c p_{i j k}=1 \\
0, \text { else }
\end{array}\right.
\end{aligned}
$$

The am-TSP can be formulated as follows:

$$
\min \sum_{k \in K} \sum_{i \in O} \sum_{j \in O}\left(c_{i j}^{T}+c_{i}\right) \cdot Y_{i j k}
$$

subject to

$$
\begin{aligned}
& Y_{i j k} \leq c p_{i j k} \\
& \sum_{k \in K} \sum_{j \in O} Y_{i j k}=1 \\
& \sum_{j \in O} Y_{i j k}=\sum_{j \in O} Y_{j i k} \\
& \sum_{j \in O \backslash O^{d e p}} Y_{i_{1} j k}=\sum_{j \in O \backslash O^{d e p}} Y_{j i_{2} k} \\
& \sum_{k \in K} \sum_{j \in O} Y_{i j k} \leq 1 \\
& \forall i \in O^{D e p_{s}} \\
& \sum_{i \in O} m_{k i}^{d}=1 \\
& \forall 1 \leq d<|D|, k \in K \\
& m_{k i}^{d} \leq \sum_{j \in O} Y_{i j k} \\
& \forall i \in O, k \in K, 1 \leq d<|D| \\
& a_{i} \leq B_{i} \leq b_{i} \\
& \forall i \in O \\
& A_{i} \leq B_{i} \\
& \forall i \in O \\
& B_{i}+t_{i} \leq D_{i} \\
& \forall i \in O \\
& D_{i}+t_{i j} \leq A_{j}+\left(1-\sum_{k \in K} Y_{i j k}\right) \\
& \forall i \in O, j \in O \\
& B_{i} \geq Q_{d}^{a}-\left(1-\sum_{k \in K} m_{k i}^{d}\right) M-\alpha_{i}^{d} \cdot M \\
& \forall i \in O, 1 \leq d<|D| \\
& B_{i}+t_{i} \leq Q_{d}^{b} \\
& +\left(1-\sum_{k \in K} m_{k i}^{d}\right) M+\left(1-\alpha_{i}^{d}\right) M \\
& \forall i \in O, 1 \leq d<|D| \\
& B T_{k}^{d} \leq D_{i}+\left(1-m_{k i}^{d}\right) M+\left(1-\alpha_{i}^{d}\right) \cdot M \\
& \forall i \in O, k \in K, 1 \leq d<|D| \\
& B T_{k}^{d} \leq B_{i}+\left(1-m_{k i}^{d}\right) M+\alpha_{i}^{d} \cdot M \\
& \forall i \in O, k \in K, 1 \leq d<|D| \\
& E T_{k}^{d} \geq A_{i}-\left(1-m_{k i}^{d}\right) M-\alpha_{i}^{d} \cdot M \\
& \forall i \in O, k \in K, 1 \leq d<|D| \\
& E T_{k}^{d} \geq B_{i}+t_{i}-\left(1-m_{k i}^{d}\right) M-\left(1-\alpha_{i}^{d}\right) M \\
& \forall i \in O, k \in K, 1 \leq d<|D| \\
& B T_{k}^{d} \geq Q_{d}^{a} \\
& \forall k \in K, 1 \leq d<|D|
\end{aligned}
$$




$$
\begin{aligned}
& E T_{k}^{d} \leq Q_{d}^{b} \\
& E T_{k}^{1}-D_{i} \leq T_{\text {max }} \\
& E T_{k}^{d}-B T_{k}^{d-1} \leq T_{\text {max }} \\
& A_{j}-B T_{k}^{|D|-1} \leq T_{\text {max }} \\
& B T_{k}^{d}-E T_{k}^{d} \geq T_{\text {min }} \\
& B T_{k}^{d}, E T_{k}^{d} \geq 0 \\
& \alpha_{i}^{d} \in\{0,1\} \\
& m_{k i}^{d} \in\{0,1\} \\
& Y_{i j k} \in\{0,1\}
\end{aligned}
$$

$$
\begin{array}{r}
\forall k \in K, 1 \leq d<|D| \\
\forall k \in K, i \in O_{k}^{\text {dep }} \\
\forall k \in K, 1<d<|D|-1 \\
\forall k \in K, j \in O_{k}^{\text {depe }_{\text {e }}} \\
\forall k \in K, 1 \leq d<|D| \\
\forall k \in K, 1 \leq d<|D| \\
\forall i \in O, 1 \leq d<|D| \\
\forall i \in O, k \in K, d \in D \\
\forall i, j \in O, k \in K
\end{array}
$$

The objective is to minimise total trucking costs (9) for the pickup and delivery of customer requests. Two nodes can only be connected if physical dimensions of nodes and vehicles are compatible (10). Each pickup and delivery node is visited once by a compatible vehicle (11). Constraints (12) imply at most one entering and one leaving vehicle for each pickup and delivery node. Constraints (13) link the start depot and end depot of each route. Only one route can depart from each start depot (14), which results in a single entering arc for each related end depot. Constraints (15) determine for each vehicle and day in the planning horizon the node at which a night's rest is included, while this can only occur if that node has an outgoing arc (constraints (16)). Time windows at each node must be respected (17)-(19). Constraints (20) set the time variables between subsequent nodes. Constraints (21)-(26) represent three pairs of constraints of which either one or the other must hold, depending on whether a service in a node is executed at any time or day before or after spending a particular night at that node. They determine the service start within daily time windows (constraints (21)-(22)), daily route start times (constraints (23)-(24)) and daily route end times (constraints (25)-(26)). Constraints (27)-(28) imply a departure after the start of day $d$ and a finish of the daily active time before the end of that day. Furthermore, a limit is placed on the time a truck can operate in a single daily route (29)-(31). The minimum night's rest between arrival and departure for a given node at which a night's rest is spent is included by means of constraints (32). Finally, the decision variables $B T_{k}^{d}$ and $E T_{k}^{d}$ are defined as positive real numbers (33), and $\alpha_{i}^{d}, m_{k i}^{d}$ and $Y_{i j k}$ must be binary (34)-(36).

\subsection{The Integrated Intermodal Container Routing Problem}

In the integrated approach, truck routing and rail planning decisions are interdependent and made simultaneously. Now, truck cost are immediately defined as the total cost of combining pickup and delivery tasks into truck routes as considered in the VRP. In the sequential problem, initially solely transport operations between each customer location and the assigned terminal are included in the long-haul routing problem, after which only the second subproblem (i.e., the VRP) considers total trucking distances.

For a single request $r$, multiple pickup tasks $O_{r}^{\text {pre }}$ and delivery tasks $O_{r}^{\text {end }}$ are available, related to the available services. A single, feasible pickup and delivery task should be performed in order to transport a request from its customer origin to its destination. A new binary variable $Z_{i}=1$ if a drayage task $i$ should be performed as a consequence of selecting the related long-haul service. All feasible drayage tasks serve as input to the problem and are defined as follows. A drayage task $i$ for pickup operations (i.e., a combination of a pickup location $g \in N^{P}$ with a rail departure terminal $h \in N^{T}$ related to available service $s$, i.e., $\lambda_{s}^{d e p}=h$ ) is feasible if the pickup location and departure terminal of a service are located in the same service region $\left(r e g_{g}=r e g_{\lambda_{s}^{d e p}}\right)$, and if a timely arrival at the delivery location $g+n$ is possible (i.e., $a_{g}+t_{g}+t_{g, \lambda_{s}^{d e p}}+t_{\lambda_{s}^{d e p}} \leq T_{s}^{d e p}$ and $T_{s}^{a r r}+t_{\lambda_{s}^{a r r}}+t_{\lambda_{s}^{a r r}, g+n}+t_{g+n} \leq b_{g}$ ). A drayage task $i$ for delivery operations (i.e., a combination of a rail arrival terminal $g \in N^{T}$ of an 
available service $s$, i.e., $\lambda_{s}^{\text {arr }}=g$, with a delivery location $h \in N^{D}$ ) is feasible if the pickup location and departure terminal of a service are located in the same service region $\left(r e g_{\lambda_{s}^{a r r}}=r e g_{h}\right)$, and if a timely arrival at the delivery location $h$ is possible (i.e., $a_{h-|R|}+t_{h-|R|}+t_{h-|R|, \lambda_{s}^{d e p}}+t_{\lambda_{s}^{d e p}} \leq T_{s}^{d e p}$ and $\left.T_{s}^{\text {arr }}+t_{\lambda_{s}^{\text {arr }}}+t_{\lambda_{s}^{\text {arr }, h}}+t_{h} \leq b_{h}\right)$. The following additional notation is used:

Decision variables:

$$
Z_{i}= \begin{cases}1 & \text { if node } i \text { is visited } \\ 0 & \text { else }\end{cases}
$$

The problem is formulated as follows:

$$
\min \sum_{r \in R} \sum_{s \in S} c_{r s}^{r a i l} \cdot X_{r s}+\sum_{k \in K} \sum_{i \in O} \sum_{j \in O} c_{i j}^{T} \cdot Y_{i j k}+\sum_{i \in O} c_{i} \cdot Z_{i}
$$

subject to

\section{Rail constraints:}

$$
\begin{array}{lr}
\sum_{s \in S} X_{r s}=1 & \forall r \in R \\
\sum_{r \in R \mid v_{r}=v} X_{r s} \leq C_{s v} & \forall s \in S_{\text {ext }} ; v \in V \\
\sum_{r \in R} l_{r} \cdot X_{r s} \leq L_{s} & \forall s \in S_{\text {own }} \\
\sum_{r \in R} w_{r} \cdot X_{r s} \leq W_{s} & \forall s \in S_{\text {own }}
\end{array}
$$

\section{Trucking constraints:}

$$
\begin{array}{lr}
Y_{i j k} \leq c p_{i j k} & \forall i, j \in O, k \in K \\
\sum_{k \in K} \sum_{j \in O} Y_{i j k}=Z_{i} & \forall i \in O \\
\sum_{j \in O} Y_{i j k}=\sum_{j \in O} Y_{j i k} & \forall i \in O \backslash O^{d e p}, k \in K \\
\sum_{j \in O \backslash O^{d e p}} Y_{i_{1} j k}=\sum_{j \in O \backslash O^{d e p}} Y_{j i_{2} K} & \forall k \in K, i_{1} \in O_{k}^{\text {Deps }}, i_{2} \in O_{k}^{\text {Depe }} \\
\sum_{i \in O} m_{k i}^{d}=1 & \forall 1 \leq d<|D|, k \in K \\
m_{k i}^{d} \leq \sum_{j \in O} Y_{i j k} & \forall i \in O, k \in K, 1 \leq d<|D| \\
a_{i} \leq B_{i} \leq b_{i} & \forall i \in O \\
A_{i} \leq B_{i} & \forall i \in O \\
B_{i}+t_{i} \leq D_{i} & \forall i \in O \\
D_{i}+t_{i j} \leq A_{j}+\left(1-\sum_{k \in K} Y_{i j k}\right) & \forall i \in O, j \in O \\
B_{i} \geq Q_{d}^{a}-\left(1-\sum_{k \in K} m_{k i}^{d}\right) M-\alpha_{i}^{d} \cdot M & \forall i \in O, 1 \leq d<|D| \\
B_{i}+t_{i} \leq Q_{d}^{b} & \\
+\left(1-\sum_{k \in K} m_{k i}^{d}\right) M+\left(1-\alpha_{i}^{d}\right) M & \forall i \in O, 1 \leq d<|D|
\end{array}
$$




$$
\begin{aligned}
& B T_{k}^{d} \leq D_{i}+\left(1-m_{k i}^{d}\right) M+\left(1-\alpha_{i}^{d}\right) \cdot M \\
& B T_{k}^{d} \leq B_{i}+\left(1-m_{k i}^{d}\right) M+\alpha_{i}^{d} \cdot M \\
& E T_{k}^{d} \geq A_{i}-\left(1-m_{k i}^{d}\right) M-\alpha_{i}^{d} \cdot M \\
& E T_{k}^{d} \geq B_{i}+t_{i}-\left(1-m_{k i}^{d}\right) M-\left(1-\alpha_{i}^{d}\right) M \\
& B T_{k}^{d} \geq Q_{d}^{a} \\
& E T_{k}^{d} \leq Q_{d}^{b} \\
& E T_{k}^{1}-D_{i} \leq T_{\max } \\
& E T_{k}^{d}-B T_{k}^{d-1} \leq T_{\max } \\
& A_{j}-B T_{k}^{|D|-1} \leq T_{\max } \\
& B T_{k}^{d}-E T_{k}^{d} \geq T_{\min }
\end{aligned}
$$$$
\forall i \in O, k \in K, 1 \leq d<|D|
$$$$
\forall i \in O, k \in K, 1 \leq d<|D|
$$$$
\forall i \in O, k \in K, 1 \leq d<|D|
$$$$
\forall i \in O, k \in K, 1 \leq d<|D|
$$

\section{Linking constraints:}

$$
\begin{aligned}
& Z_{i}=X_{r \sigma_{i}} \\
& X_{r \sigma_{i}}=Z_{i}
\end{aligned}
$$

$$
\begin{aligned}
& \forall r \in R ; s \in S ; i \in O_{r}^{\text {pre }} \\
& \forall r \in R ; s \in S ; i \in O_{r}^{\text {end }}
\end{aligned}
$$

\section{Domains:}

$$
\begin{aligned}
& B T_{k}^{d}, E T_{k}^{d} \geq 0 \\
& \alpha_{i}^{d} \in\{0,1\} \\
& m_{k i}^{d} \in\{0,1\} \\
& X_{r s} \in\{0,1\} \\
& Y_{i j k} \in\{0,1\} \\
& Z_{i} \in\{0,1\}
\end{aligned}
$$

$$
\begin{array}{r}
\forall k \in K, 1 \leq d<|D| \\
\forall i \in O, 1 \leq d<|D| \\
\forall i \in O, k \in K, d \in D \\
\forall r \in R ; s \in S \\
\forall i, j \in O, k \in K \\
\forall i \in O
\end{array}
$$

The objective is to minimise total trucking and rail costs (37) for the delivery of customer requests. Constraints (38)-(41) relate to (2)-(5) of the intermodal routing problem, where one of the feasible services for each request must be selected (with for each feasible service, one feasible pickup and one feasible delivery task). Trucking operations are modelled by means of constraints (42)-(63), related to constraints (10)-(32). As multiple pickup and delivery tasks are often available for each request $r$, a single pickup node $i \in N_{r}^{\text {pre }}$ and delivery node $i \in N_{r}^{\text {end }}$ should be visited, which is implicitly included. The link between truck route times and rail departures and arrivals are included in the definition of the nodes or tasks. Precedence constraints (64)-(65) link a single pickup and delivery task per request to its related rail service. Finally, the decision variables must be positive real numbers (66), or binary (67)-(71).

\section{Large Neighbourhood Search Heuristic Solution Method}

A large neighbourhood search (LNS) [46] algorithm is developed to solve the intermodal routing, in which in each single iteration a large number of requests is removed, and next reinserted into the solution. The framework has proven to lead to good results for vehicle routing problems and pickup and delivery problems [47]. Both a sequential and an integrated version of the heuristic algorithm are presented. The sequential approach serves as a benchmark for the improvements that may be obtained by the integrated intermodal routing problem. The heuristic building blocks are similar for both approaches, but different parts of the solution are destroyed and repaired. For each request, one pickup drayage task (from a customer pickup location to a departure terminal) and one delivery drayage task (from an arrival terminal to a customer delivery location) have to be executed. Both tasks relate to the 
selected long-haul service. Whereas operators in a sequential setting remove and reinsert individual, fixed drayage tasks (with predetermined terminals), an integrated setting removes and inserts both the pickup and delivery task of any request simultaneously, as well as the selected long-haul service.

Moreover, in practice, the planning for one time period is executed, while an initial planning for future time periods is included, to be used as a starting point for the following time period. We pay attention to this dynamic nature of the problem by including information from past week's planning as well as the initial planning for the following week. If this information would be excluded, besides the fact that this is unrealistic, many pickup tasks would occur at the beginning of the current week, and many delivery tasks would need to be executed at the end of the week. Moreover, this would result in a limited choice of long-haul services, with a lower capacity utilisation for long-haul services with departure in the beginning and at the end of the week, compared to long-haul services in the middle of the week. First, we include requests which were already assigned to a long-haul service with departure in the previous week. For these requests, only the truck delivery task still has to be executed after arrival at the destination terminal. In practice, this is given input for current week's planning. Therefore, it is assumed that these requests are assigned to long-haul services in advance, and only the delivery trucking cost is relevant for this week's problem. The assigned long-haul service and related delivery task do not vary during the execution of the heuristic. The delivery task should only be assigned to a position in a truck route. Second, some requests are assigned to long-haul services which depart in the current planning cycle and arrive in the next. For this type of assignment, the long-haul service is selected by the heuristic. Only the pickup task should be included in truck routes. Delivery tasks in the next week are included by means of the two-way direct distance between the destination terminal and the customer destination, plus a high value $M$ in order to avoid postponing requests. At the same time, this ensures that if postponement is required, a smaller end-haul trucking distance is preferred. For these requests, total assignment costs consist of costs for including the pickup task in truck routes (VRP), long-haul service costs, and direct two-way end-haul truck costs.

The general heuristic structure is presented in Section 4.1, after which the removal and insertion operators are described in Section 4.2. Preliminary feasibility checks for inserting a single node into a candidate solution are explained in Section 4.3. If these checks do not indicate an infeasible solution, a more complex, multi-day scheduling procedure aims at finding a feasible truck schedule for the candidate solution. The procedure is discussed in detail in Section 4.4. These checks are performed in this sequence, with the least time-consuming checks first, as it is hard to determine in advance whether a solution is feasible in a multi-day scheduling context with a minimum overnight's rest and a maximum daily active duration.

\subsection{General Structure of the LNS}

Algorithm 1 provides the general heuristic structure. It includes a constructive heuristic (lines 2-4) as well as a framework with several removal operators (lines 9-12) and insertion operators (lines 13-15).

The constructive heuristic consists of first assigning requests to long-haul services by solving the long-haul routing problem using integer linear programming using ILOG Cplex 12.6 in $\mathrm{C}++$, and next inserting for each request its pickup and delivery task-related to the known, assigned long-haul services-into truck routes. These given drayage tasks are inserted in a random order at their best feasible insertion position, i.e., with the lowest additional cost, in order to obtain a good initial solution. This corresponds to the random order best insertion operator of the sequential approach.

In each iteration, one removal operator is selected randomly in which requests or tasks are removed until a given threshold is reached with respect to the number of removed requests or tasks. Next, one insertion operator is selected randomly. Requests are reinserted in order to obtain a new, complete solution. In a sequential approach (Seq.), after requests are assigned to long-haul services in the constructive phase, minimum-cost truck routes are established with known pickup and delivery tasks. Sequential operators aim at removing or inserting single drayage tasks, instead of requests, 
and the assigned long-haul services remain unchanged. In an integrated approach (Int.), destroy and repair operators consider removing and reinserting requests from the solution. More information on these integrated operators is presented in Section 4.2.

The acceptance criterion for moves includes a function with multiple cost components (lines 18-20). The total costs of each solution consist of vehicle routing costs in both service regions (sol new. vrp Cost), two-way direct truck costs for drayage tasks to be executed in the next planning period as a result of long-haul services with arrival in the next week (sol new. directTruckCost), and long-haul transport costs (sol $l_{\text {new }}$.railCost). Moreover, for accepting new solutions, a worse solution is allowed to be accepted for the vehicle routing cost (solnew.vrpCost $\cdot(1+$ deviation $)$ ) in order to focus on the optimisation of vehicle routes in each service region.

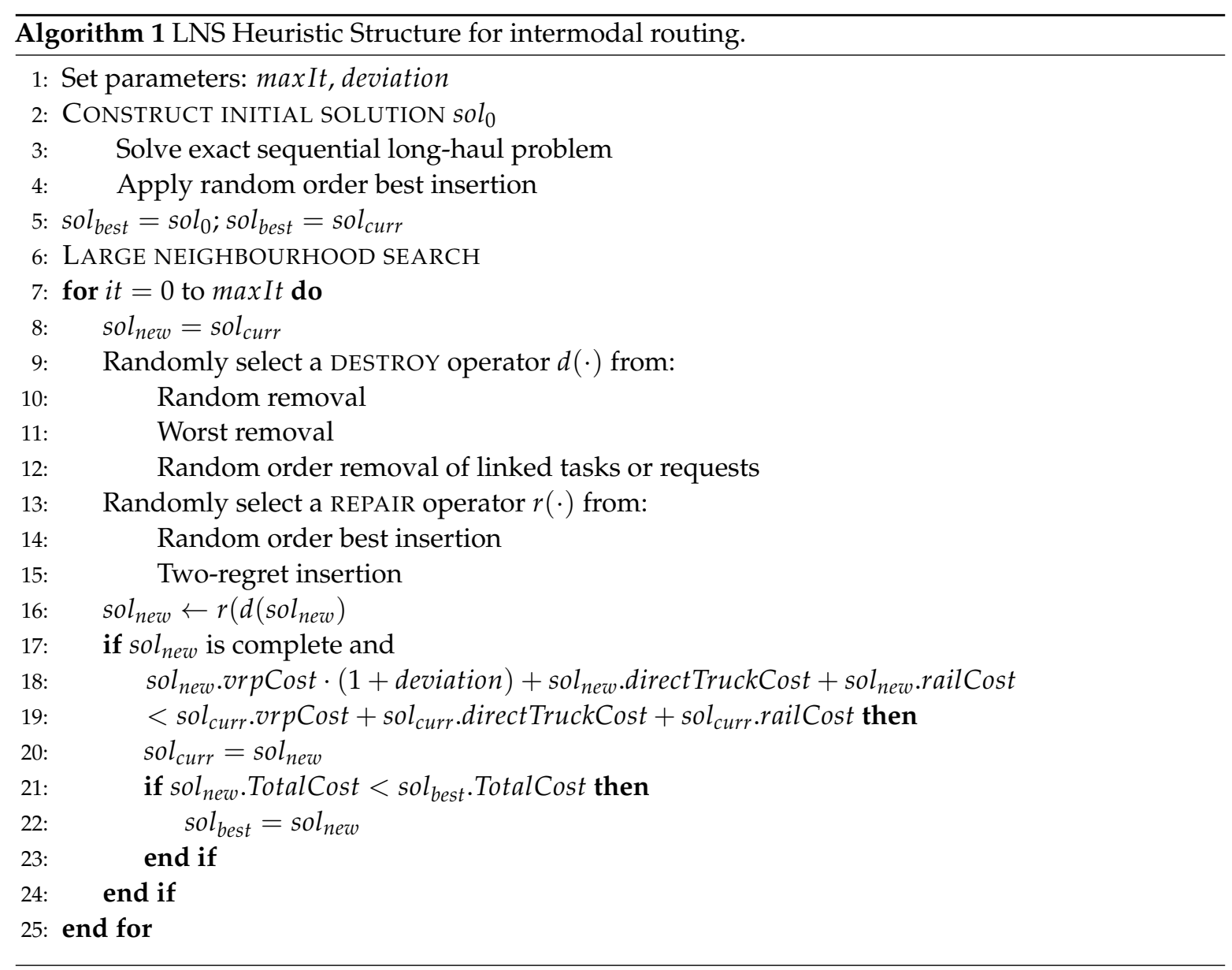

\subsection{Integrated Operators}

In this section, the operators used within the large neighbourhood search framework are described. Operators which are well-known and have shown to obtain good results for pickup and delivery problems (random and worst removal, random order best insertion and two-regret insertion) [47], as well as an operator designed to fit the characteristics of the problem are included (random order removal of linked tasks/requests). Integrated operators, which are dedicated to the integrated nature of the problem, are explained in this section. Sequential operators are not discussed individually, but use the same building blocks. The only difference is that in the sequential approach, improvements in truck routes are considered without changing selected long-haul services, as this decision is made in advance. To summarise, sequential operators focus on individual drayage tasks, whereas integrated operators address the entire intermodal route of a request, i.e., the pickup and delivery task as well as the assigned long-haul service. Four removal operators and three insertion operators are included and 
described next. The removal procedure is repeated until the number of removed requests or drayage tasks, for the integrated and sequential approach respectively, reaches a given threshold. The process of inserting uninserted requests or drayage tasks is repeated until either all requests are inserted or no feasible insertion can be found for a request. If no feasible insertion can be found, the solution is incomplete and the iteration ends.

Random removal of requests: Randomly selected requests are removed from the solution until a given threshold is reached.

Worst removal of requests: Requests are ordered based on the savings that could be obtained by excluding that request from the solution, i.e., if its pickup task and delivery task are omitted from truck routes and the request is removed from the previously assigned long-haul service. The request with the largest savings is removed and savings are updated.

Random order removal of linked requests: Randomly selected requests are removed from the solution. For each randomly selected request $r$, both the pickup and delivery task are removed, and the request is removed from its assigned long-haul service. Further, if any delivery task immediately precedes the pickup task of request $r$ in its current truck route, the request related to that delivery task is removed too. Similarly, if any pickup task immediately succeeds the delivery task of request $r$, the request related to that pickup task is removed. Obviously, in the sequential variant of this operator, if a pickup task is selected, the delivery task executed right before is removed if available. For selected delivery tasks, a single pickup task executed afterwards is removed, again if available. By removing subsequent pickup and delivery tasks, subroutes (or street turns) between two terminal visits can be removed from the solution.

Random order best insertion of requests: Uninserted requests are selected randomly. For each request, the best long-haul service is selected, defined by taking an integrated perspective. The sum of the long-haul rail cost and the best corresponding pre- and end-haul cost for inserting tasks into truck routes determine the total cost of assigning a request to a long-haul service and inserting it into a route in each region.

Two-regret insertion of requests: First, for all uninserted requests, the best and second-best insertion costs are calculated, defined by the best and second-best long-haul service, insertion of the pickup node and insertion of delivery node, again taking an integrated perspective. Next, the request with the highest difference between the lowest and the second-lowest total insertion cost is inserted in the solution, after which insertion costs for the remaining requests are updated if required. The idea is to first insert the requests which are the most difficult to insert in order to avoid that they cannot be inserted feasibly anymore if more requests are inserted.

Two other operators, a removal of related requests from similar services and a greedy insertion were considered. However, experiments showed that their omission caused a better objective value. As the removal of both operators simultaneously also results in a significantly increased solution quality, the sequential and integrated heuristic without the greedy insertion and related removal operator will be used in the remainder of this paper.

\subsection{Preliminary Checks for Inserting a Node}

Inserting a node $n$ at its best insertion position, i.e., after predecessor $i$ currently assigned to a route, can only be feasible if several conditions hold. These conditions focus on a single insertion. First, only insertion positions within the same service region are considered $\left(r e g_{i}=r e g_{n}\right)$. Next, an initial check on the time windows examines whether $a_{i}+t_{i}+t_{\text {in }} \leq b_{n}$ between a predecessor $i$ and $n$, and whether $a_{n}+t_{n}+t_{n j} \leq b_{j}$ for inserting $n$ before the current successor of $i$ (denoted with $j$ ). If also the dimensions of both nodes match $\left(l_{i}=l_{n}\right)$, it is checked whether a node $n$ can be feasibly inserted between node $i$ and its current successor $j$ based on a regular calculation of the earliest start $E S$ (forward from predecessor $i$, with $E S_{n}=\max \left\{a_{n} ; E S_{i}+t_{i}+t_{i n}\right\}$ ) and latest start $L S$ (backward from the current successor of $i$, with $\left.L S_{n}=\min \left\{b_{n} ; L S_{j}-t_{n j}-t_{n}\right\}\right)$, respecting its time window $\left[a_{n}, b_{n}\right]$. 


\subsection{Feasibility of New Routes}

Only if all conditions in the preliminary check are satisfied (see Section 4.3)—which are required, but insufficient conditions for feasibility-, another procedure determines whether a feasible schedule can be found for a certain truck with a given sequence of nodes in a candidate solution. As trucks can be out for multiple days, it would not be realistic to assume that they are active for an entire week. Therefore, the scheduling procedure for multiple days should include a minimum overnight's rest and a maximum daily active time. The day at which a task should be executed is not fixed in advance (within given release and due times of a request) and, as such, is part of the decision process.

Nodes in a given sequence are added to a given day's route using the earliest feasible start as long as the daily limit on the route duration $T_{\max }$ is not violated. If not feasible, the forward time slack principles, explained in detail later on, are used to shift the route schedule within a single day. It is always better to travel to and serve a node on the current day than shifting one or both of these activities to a future day. Therefore, if a feasible route can be composed on a particular day by reducing that day's route duration, and thus postponing the start of that route, this is always executed. In this part of the procedure, the earliest possible arrival in the last node of the day is assumed to be given and does not shift. Hence, the shift does not restrict the feasibility of the next day. Only if shifting the start of the route is not feasible, travel to and/or service of the node under consideration is tried to be performed on a future day and the entire procedure is repeated for that future day. If all nodes in a route can be feasibly visited, the procedure provides a schedule which satisfies a maximum daily route duration $T_{\max }$, a minimum night's rest $T_{\min }$ and daily time windows $Q_{d}^{b}$ and $Q_{d}^{a}$ between which trucking is allowed.

Within this procedure, four types of feasible scheduling options are considered for each node to be added to a route. These are explained below. The procedure evaluates a given sequence of nodes, where nodes $i$ and $j$ are assumed to be two subsequent nodes in the sequence. If adding a new node $j$ does not result in any infeasibility, the next node in the sequence will be evaluated (i.e., $i=j$ and $j=$ successor $_{j}$, and the procedure is repeated).

The procedure requires two modifications to the forward time slack $(F)$ principles of Savelsbergh [48] and Vidal et al. [49]. The forward time slack $(F)$ indicates the time with which each node served on a particular day can be shifted later in time such that a feasible route can be composed serving all nodes before (and including) the currently considered node based on the earliest start procedure, which calculates the earliest feasible start of a task at any node in a sequence using time windows, service times and travel times. To calculate the forward time slack, both the earliest feasible execution date $T_{i}$ and the cumulative idle time $W_{i}$ are required for a given node $i$. A first modification, $F *_{\text {service }}$ is explained in case 2 below. A second modification, $F *_{\text {noservice }}$ is used in case 3 .

Case 1: Travel and service on current day $d$ without changing service start of nodes in route of current day. If adding travel to and service of node $j$ to the current day's route results in a feasible route duration (i.e., $T_{\text {service }} \leq T_{\max }$, with $T_{\text {service }}$ equal to the route duration of the current day) and if it can be performed before the end of the current day $Q_{d}^{b}$, node $j$ is added to the current day's route if this does not result in any infeasibilities of the route of future nodes.

Case 2: Travel and service on current day $d$ with removal of excess waiting time in route of current day. If adding a node $j$ with the regular earliest feasible start results in a violation of the maximum daily route duration $\left(T_{\text {service }}>T_{\max }\right)$, it is checked whether it is possible to postpone the service start of previous nodes in the route of the current day such that node $j$ can be feasibly included on the current day's route within the maximum daily route duration. If feasible, this is always preferred compared to serving it on a future day. In this case, a first modification to the regular forward time slack $F *_{\text {service }}=\min \left\{F_{i}, W_{j}\right\}$ is performed. Using the regular time slack $F$ could influence the start of the minimum overnight rest and consequently the start time of the next day, which might lead to an infeasible schedule on the next day. Therefore, one difference with the regular time slack principles is the fact that the service start of the currently considered node $j$ remains unchanged when attempting to shift the start of a day's route. To feasibly include travel to and service at node $j$, the maximum daily 
route duration should be respected after shifting the current route $\left(F *_{\text {service }} \geq T_{\text {service }}-T_{\max }\right)$, and the truck should be able to finish service before the end of that day $\left(E S_{j}+t_{j} \leq Q_{d}^{b}\right)$. Figure 3 visualises such a feasible shift. Initially, inserting node $j$ is infeasible with a given start of the day, in this case represented by the start of service in node $i$. However, by postponing the start of a route on a given day with $F *_{\text {service, }}$ it becomes feasible to travel to and serve node $j$, without changing its earliest feasible service start. At the same time, excessive wait time on the route of the current day is reduced.

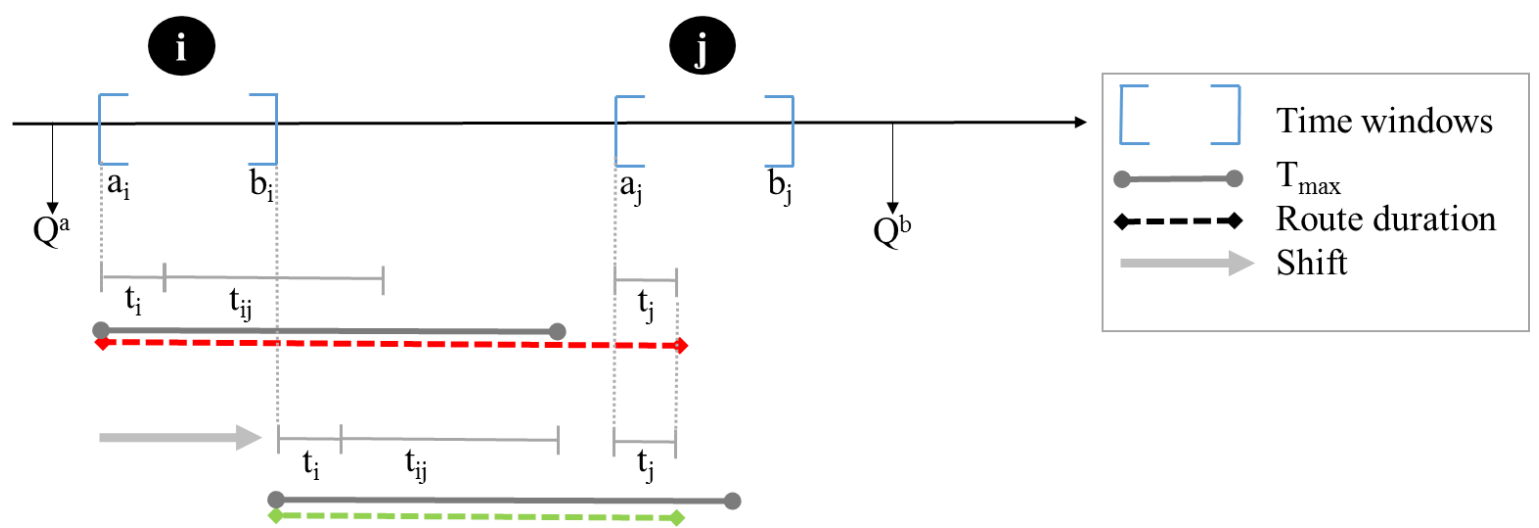

Figure 3. Shift the start of a route on a single day with $F *_{\text {service, }}$ including travel to and service of node $j$ on the current day.

Case 3: Travel on current day $d$ with removal of excess waiting time in route of current day, service on a future day. If adding a node $j$ with the current earliest feasible start results in a violation of the maximum daily route duration (i.e., case 2 is infeasible), it is checked whether it is possible to postpone the service start of previous nodes in the route of the current day such that the travel to node $j$ can be feasibly included on the current day within the maximum daily route duration. An important aspect within this regard is the fact that a minimum night's rest might need to be added before or after serving (i.e., travel towards a node can occur on that day, before actually serving that node on a future day). However, a truck is only allowed to rest with an empty container. Therefore, if postponing the route with $F *_{\text {service }}$ is infeasible, a second modification to the forward time slack $F *_{\text {noservice }}=\min \left\{F_{\text {predec }_{i}}, W_{i}\right\}$ is performed, which is equal to zero if no predecessor of $i$ is available on the current day (i.e., no node is served before this node on the current day). It is used to check whether it is feasible to only include the travel to node $j$ on the current day, while service occurs on any future day. Furthermore, the service start of the last node on the current day (represented by node $i$ in Figure 4) is not allowed to shift, as this could influence the feasibility of the next day's route. Again, three conditions must hold: the current day should not be the last day in the planning horizon $(d<D)$, the current day's route can be feasibly shifted such that it does not exceed the maximum daily active time $\left(F *_{\text {noservice }} \geq T_{\text {noservice }}-T_{\max }\right)$, where $T_{\text {noservice }}$ denotes the total active time of a truck on the current day with travel to but without service of node $j$, and travel should be finished before the end of the current day $\left(T_{i}+t_{i}+t_{i j} \leq Q_{d}^{b}\right)$.

If a single overnight's rest is required before service at node $j$ occurs on the following day, $\max \left\{E S_{j} ; \max \left\{Q_{d^{\prime}}^{a} T_{i}+t_{i}+t_{i j}+T_{\min }\right\}\right\}$ defines the earliest start. If more than one day passes between travel to and service at node $j$, the service start is calculated by $\max \left\{Q_{d}^{a}, T_{j}\right\}$. If the service start resulting from this procedure turns out to be infeasible, the solution is infeasible.

Case 4: Travel on next day $d+1$, service on future day $\geq d+1$. If cases 1,2 and 3 are not feasible, it is tested whether it would be feasible to include a night's rest after serving node $i$ on day $d$. Travel to node $j$ is performed on the next day $d+1$ and service of node $j$ occurs on any future day $d \geq d+1$. If travel and service can be feasibly inserted on the same future day, after a minimum night's rest, the service start is defined by $\max \left\{Q_{d}^{a}+t_{i j}, E S_{j}\right\}$ if this is on the next day, or by $\max \left\{E S_{j}, \max \left\{Q_{d}^{a}+t_{i j} ; T_{i}+t_{i}+T_{\min }+t_{i j}\right\}\right\}$ any day after the next. Identical to case 3 , if one or 
more days pass between travel and service, the service start of node $j$ as a first activity on any day further in the future depends on the number of days that pass between travel to and service of node $j$.

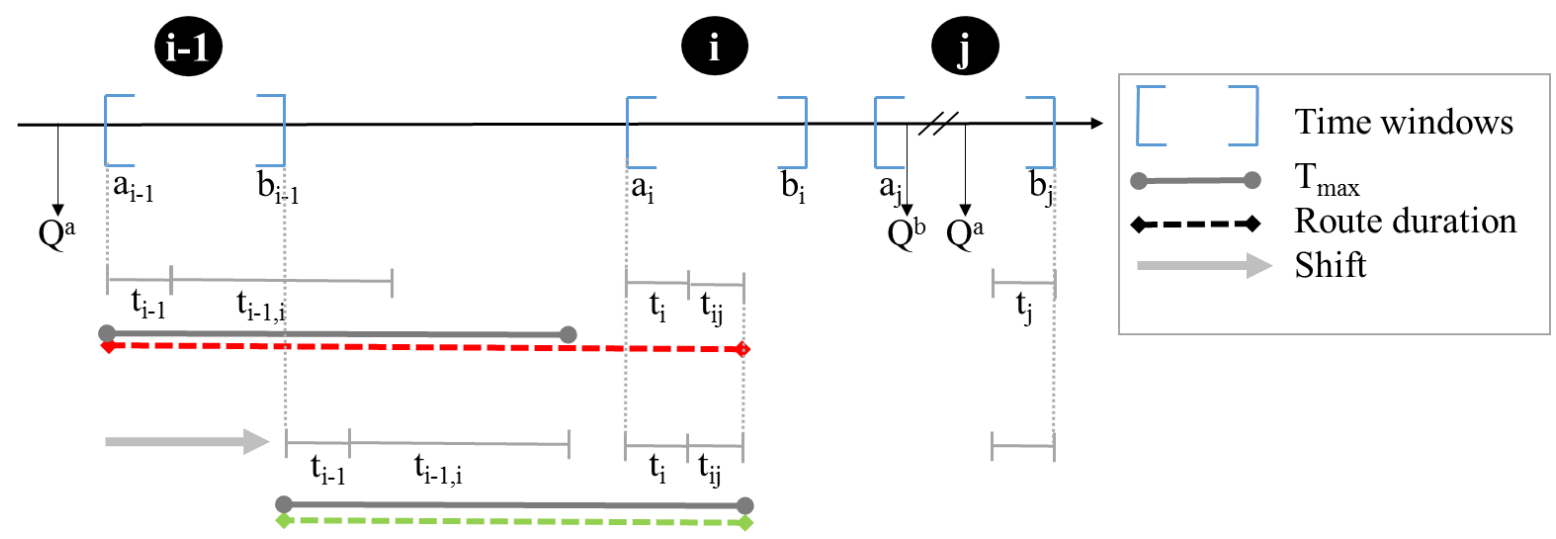

Figure 4. Shift the start of a route on a single day with $F *_{\text {noservice, }}$ including travel to node $j$ on the current day, and service of $j$ on a following day.

\section{Comparison of the Sequential and Integrated Approach}

The large neighbourhood search heuristic is used to compare results of a sequential and integrated approach. Realistic instances (confidential company data) based on a real-life case study are used as input. An experimental design is proposed to determine an appropriate parameter setting. With these parameter values, the added value of the integrated approach is demonstrated. The experiments are conducted on an Intel Xeon E5-2680v3 processor at $2.5 \mathrm{GHz}$ of the Flemish Supercomputer Center (VSC).

\subsection{Generated Instances}

A real-life case study is investigated in order to provide realistic input to the problem. In the case study, the intermodal operator offers direct rail connections between multiple terminals located within two service regions, the Benelux and Northern Italy. Trains are operated based on given periodical schedules with a cycle of one week, using both own trains and slots on trains operated by other companies. For own trains, the available loading metres and path weight restrictions limit the flows on each route, while for long-haul services managed by other parties the number of slots per length type and their weights must be accounted for. The costs for using long-haul services are fixed once available long-haul services are known.

Long-haul services: The long-haul rail network in this case study consists of real-life long-haul services between two service regions with both forward and backward flows between the regions. This results in 84 unique long-haul services in total, of which multiple long-haul services could consist of the same connection between two terminals, with different departure times. All service characteristics, such as the capacity, departure time, travel time and arrival time of each service are based on real-life data of weekly rail services. The terminals are located at their real-life locations, four within each of the two service regions of $300 \mathrm{~km}$ by $250 \mathrm{~km}$ (Figure 5). One terminal per region is selected as a truck depot, and a sufficient number of trucks is assumed to be available at each depot. Trucking distances are calculated by means of the euclidean distance between two points.

Requests: Pickup and delivery locations for a request are either defined randomly or clustered within each square service region. Clustered request locations are obtained using intermodal terminals as cluster centres based on the procedure explained by Cordeau et al. [50], with phi $=0.08$. By considering both clustered and random customer locations, the influence of customer locations can be analysed. Moreover, the demand level is varied between a relatively low and high capacity utilisation, with a maximum long-haul capacity of 470 requests for all long-haul services with departure in a single week (and arrival this week or the next). It corresponds to a scale of 1:3 in comparison with 
real-life problems. Additional requests are generated to account for last week's planning. Requests should be picked up and delivered at customer locations within 5 days after their release date. It is assumed that requests are released uniformly over the extended planning horizon. An equal demand of two types of container requests and trucks ( $30 \mathrm{ft}$ and $45 \mathrm{ft}$ ) is assumed. The variation in demand characteristics results in $2 \times 3$ instance classes, with either randomly generated or clustered customer locations and the demand (in number of requests to be transported) set to either $75 \%, 85 \%$ or $95 \%$ of the overall long-haul service capacity. The case with $95 \%$ capacity utilisation relates the most to the circumstances a real-life intermodal operator deals with in practice.

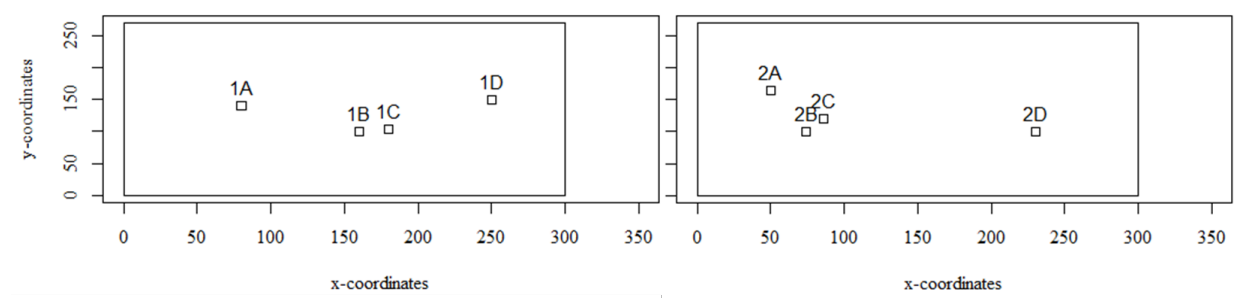

Figure 5. Terminals in two service regions.

All combinations of input characteristics for the customer locations and demand level are considered, resulting in six instances classes. An overview is provided in Table 1.

Table 1. Instance classes for intermodal routing.

\begin{tabular}{ccc}
\hline Class & Locations & Demand Level \\
\hline 1 & random & $75 \%$ \\
2 & cluster & $75 \%$ \\
3 & random & $85 \%$ \\
4 & cluster & $85 \%$ \\
5 & random & $95 \%$ \\
6 & cluster & $95 \%$ \\
\hline
\end{tabular}

Other input: Values for remaining input parameters are summarised in Table 2. Depot opening hours $\left[a_{g} ; b_{g}\right], g \in N^{d e p}$ are set to the length of current week's planning horizon. Service times for pickup and delivery operations at customers $t_{g}, g \in N^{P}, N^{D}$, and load and unload operations at terminals $t_{g}, g \in N^{T}$ are added. Service time at the depot $t_{g}, g \in N^{D e p}$ is assumed to be zero, as it is assumed that no operations occur at the depot. A trucking $\operatorname{cost} c_{i j}$ of one Euro per kilometre travelled is assumed.

Table 2. Input parameters for intermodal routing.

\begin{tabular}{cc}
\hline Input Parameter & Value \\
\hline$\left[a_{g} ; b_{g}\right], g \in N^{d e p}$ & {$\left[0 ; 6^{*} 24\right] \mathrm{h}$} \\
$t_{g}, g \in N^{P}, N^{D}$ & $2 \mathrm{~h}$ \\
$t_{g}, g \in N^{T}$ & $1 \mathrm{~h}$ \\
$t_{g}, g \in N^{D e p}$ & $0 \mathrm{~h}$ \\
$T_{\max }$ & $15 \mathrm{~h}$ \\
$T_{\min }$ & $6 \mathrm{~h}$ \\
$c_{i j}$ & 1 euro per $\mathrm{km}$ \\
\hline
\end{tabular}

\subsection{Heuristic Parameters}

The goal of this research is to explore and quantify the differences between a sequential and integrated approach. Within this aim, the parameter setting should allow a fair comparison of both approaches. Therefore, for each solution approach, the best parameter setting is determined 
independently. This implies that the parameter settings for the sequential and the integrated approaches may differ.

For the purpose of tuning the heuristic parameters, two instances are generated per instance class. The result is an experimental design with 12 instances as input, with three factor levels for the removal percentage when destroying part of the solution, and four factor levels for the allowed percentage deviation from the best solution in order to accept new moves. All combinations are tested. Five replications are performed for each instance, for each combination of parameter values. Each replication of a single instance is tested using both approaches. Experimental results executed to determine the parameter setting are provided in Appendix A. Parameter values (Appendix A.1) as well as the stopping criterion (Appendix A.2) are analysed. A summary of the selected parameter values for each approach can be found in Table 3.

Table 3. LNS parameter setting for testing.

\begin{tabular}{ccc}
\hline Input Parameter & Sequential & Integrated \\
\hline Number of iterations & 3000 & 3000 \\
Acceptance threshold of new solutions $d e v$ & $0 \%$ & $1 \%$ \\
Removal of requests & $5 \%$ & $5 \%$ \\
\hline
\end{tabular}

\subsection{Experimental Results}

To compare the results of both approaches, experiments are performed with the defined parameter setting using new instances with identical demand characteristics. Within each of the six instance classes, ten instances are generated. Five replications are performed for each instance. Figures 6 and 7 summarise the results obtained for the sequential (in grey) and integrated (in blue) intermodal routing heuristic for all demand characteristics.

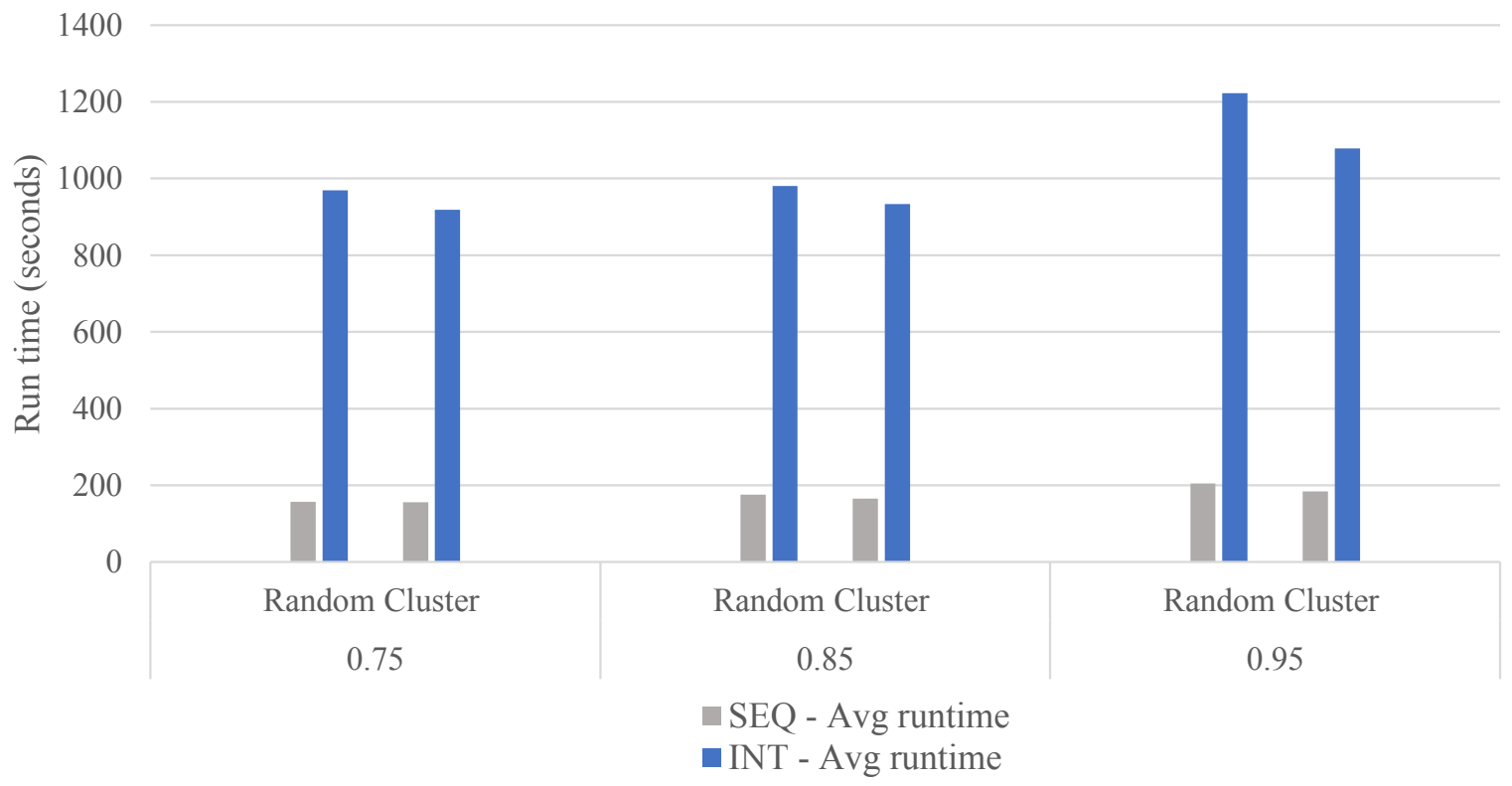

Figure 6. LNS results: computation times for the integrated and sequential approach.

Generally, the sequential approach leads to shorter run times (Figure 6), which is due to the fact that drayage tasks are considered separately. Drayage tasks in different service regions are known and planned independently, as the long-haul service is unchanged. On the other hand, with a focus on changing the route of entire requests in the integrated viewpoint, a larger computation time is required to find a feasible insertion for both the pickup and delivery drayage task when considering assigning that request to another long-haul service. 


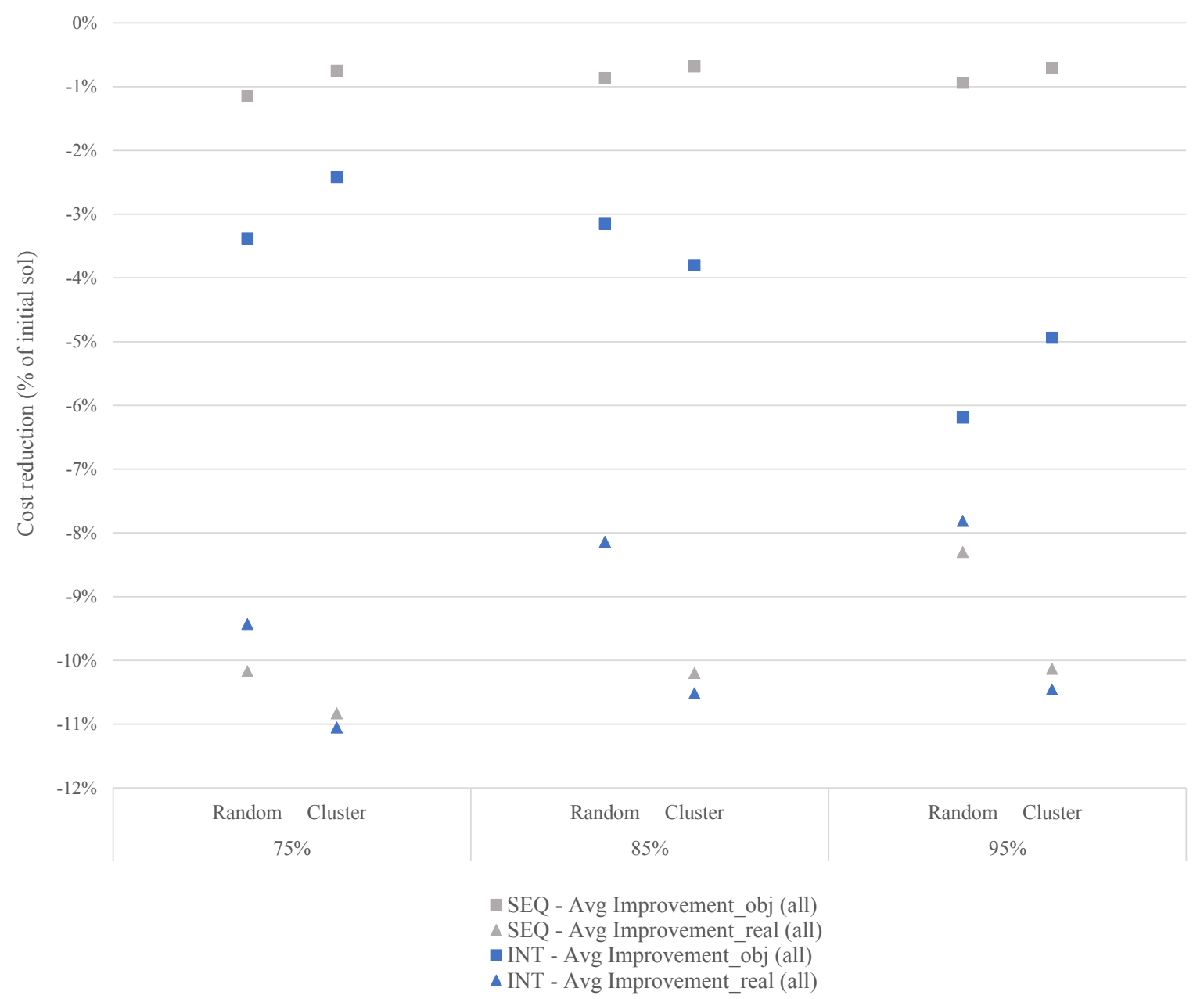

Figure 7. LNS results: solution quality for the integrated and sequential approach.

In Figure 7, the reduction in the objective value (compared to the initial solution of the constructive heuristic) and total real cost are displayed by means of rectangles (improvement_obj) and triangles (improvement_real) respectively. The lower on the vertical axis, the better. The improvement in the real total costs shown in blue (improvement_real) are based on the real total costs incurred, i.e., the sum of long-haul service costs for all departures in the current planning week, costs of truck routes in the current planning week, and direct two-way trucking costs for delivery tasks in the future week (excluding the artificially added high value for assigning requests to long-haul services with arrival in the next week).

With respect to the objective function value (Improvement_obj), few improvements are obtained in the sequential approach, as long-haul services are already fixed and known. Cost reductions are due to a reduction in drayage costs. The objective value is clearly lower in an integrated approach, which demonstrates the advantage of the integrated approach. It is partly due to the fact that more load units can be assigned to long-haul services in the current planning week.

As illustrated by the improvement in the real total cost (Improvement_real), the integrated approach seems especially promising for instances with clustered demand locations. More load units can be planned in the current planning cycle at a reduced total cost. By introducing flexibility in the long-haul service, other drayage tasks can be considered which provide more opportunities to further reduce trucking costs in the service regions. For instances with random customer locations, more load units can be planned, at the cost of an increased drayage cost. Please note that costs are identical for the $85 \%$-random-case. For a smaller demand level, the closest terminal can always be selected if enough capacity is available, and no trade-offs are required. On the contrary, with a higher 
capacity utilisation, a decision should be made based on the relationship between costs of truck routes and assigned long-haul services, and the closest terminal cannot always be selected. In all demand scenarios, the integrated approach postpones fewer load units to next week's long-haul services at a reduced or small additional truck cost.

Comparing both approaches, the difference in the number of load units assigned to a long-haul service with arrival and departure in the current planning cycle can be observed from Figure 8 . Values larger than zero indicate a higher number of load units assigned to this week's long-haul services in the integrated approach compared to the sequential approach.

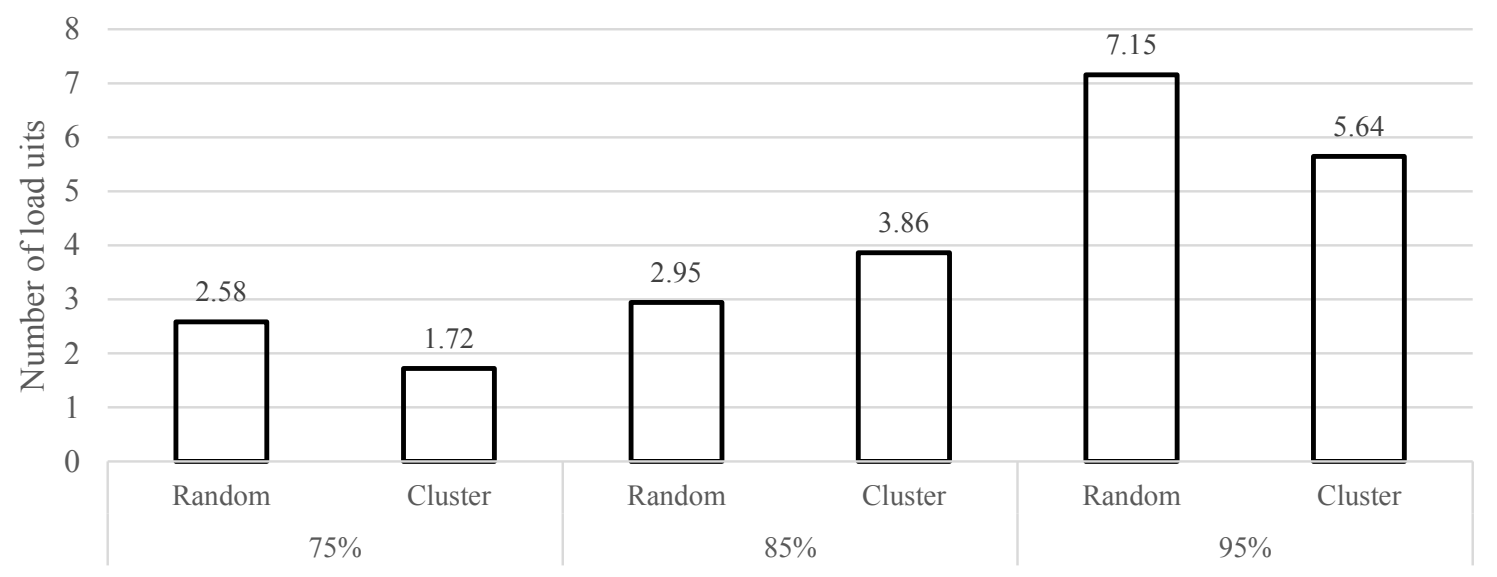

Figure 8. Average difference in the number of load units planned with departure and arrival in the current planning week.

Clearly, as already suggested based on the general results, for all demand scenarios, the integrated approach is able to process more requests in the current planning cycle. These differences can be explained by the fact that no feasible insertion to long-haul services with departure and arrival in the current planning week is obtained for some requests in the construction of the initial truck routes. Whereas in a sequential viewpoint, no change to the assigned long-haul service is considered, the integrated approach is able to find an assignment to a long-haul service with arrival in the current planning week.

Figures 9 and 10 display the average absolute and percentage difference in the cost per drayage task between the sequential and the integrated approach. A positive value indicates a reduced cost for the integrated approach. The measures are calculated under the assumption of one Euro per euclidean distance unit.

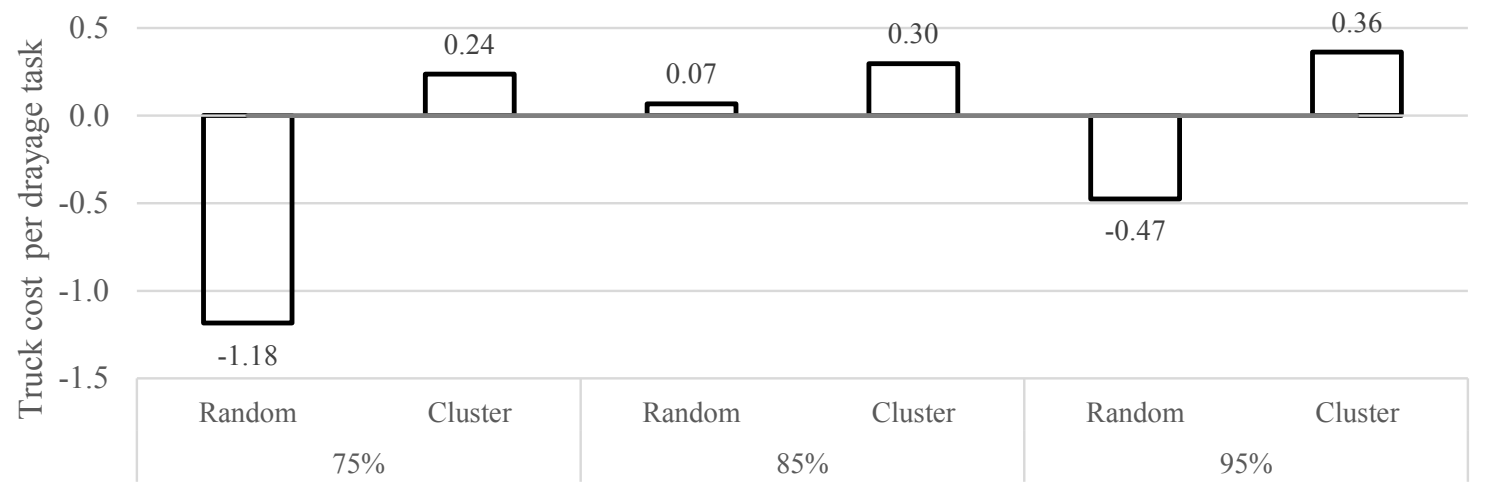

Figure 9. Average absolute difference in the cost per drayage task. Positive values indicate a lower cost in the integrated approach. 


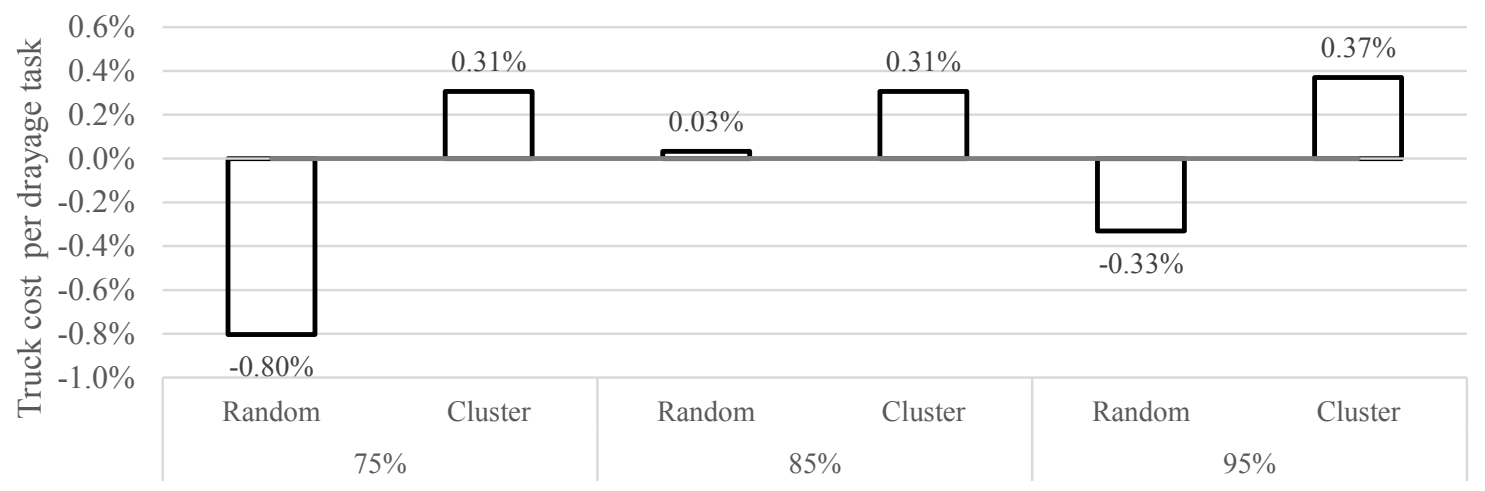

Figure 10. Average relative difference in the cost per drayage task. Positive values indicate a lower cost in the integrated approach.

Results show a lower trucking cost per drayage task for instances with clustered customer locations in an integrated approach compared to a sequential approach. The higher the capacity utilisation, the larger the savings. On the one hand, a cost reduction is obtained by providing more efficient truck routes. On the other hand, this effect is reinforced because of the fact that more load units are planned on a long-haul service which departs and arrives in the current planning cycle. This further decreases costs as this higher number of load units can also be planned more efficiently.

For demand with random customer locations, it becomes harder to combine drayage tasks in existing truck routes, as distances between the locations are relatively larger. This is also reflected in the results by means of increased costs. Some customer locations might be relatively far away from some terminals, which makes the terminal selection a fixed choice. In these cases, the sequential approach is able to focus on improving truck routes with the given drayage tasks, whereas the integrated approach does not explicitly deal with optimising the truck routes for given drayage tasks. Therefore, the sequential approach might be able to include tasks which are harder to insert into truck routes in a more efficient way.

Trade-offs might exist between increasing the capacity utilisation in the current planning week and reducing trucking costs. For some load units, it could be possible to include these in this week's planning. However, in order to plan these orders, a higher trucking cost must be incurred, especially if the demand is spread randomly across the service region.

To conclude, results of the integrated approach demonstrate that the largest savings are obtained for clustered instances with demand characteristics closest to real-life cases (i.e., high capacity utilisation). In all cases, fewer load units are postponed, consistent with an improved capacity utilisation. For instances with high demand, trucking costs per drayage tasks are reduced in the case of clustered instances, but not for random customer locations.

\section{Case Study: Tactical Service Network Design Decisions}

Using the integrated approach for real-life data, insights can be obtained into how operational transport costs are influenced by decisions concerning the service network design at the tactical level. This section serves as an exploratory study on the impact of small changes in the service network on operational costs.

In the medium term, decisions on the service network design are usually determined based on expected aggregate demand flows, without knowledge of their impact on the operational planning. Besides, the decision on the offered service network may also be of strategical importance for reasons of competitiveness. This results in sunk costs for operating long-haul services (e.g., wagon leases, costs of purchased slots) in the short term, in addition to variable transport costs. The analyses in this section aim to demonstrate an additional way in which the integrated intermodal routing problem can be used in practice. Intermodal rail service operators can use this modelling approach to optimise 
the utilisation of their service network and adapt their services to their customers' expectations by changing some of their decisions in the future, for example when renegotiating transport contracts of slots on long-haul services.

The effect of two possible scenarios impacting the service network is studied in order to analyse their impact on the total operational transport costs of the system. Section 6.1 examines the possible advantages of dividing the overall long-haul rail capacity over a smaller number of long-haul services with a higher capacity per departure. Section 6.2 analyses the impact of congestion at and around terminals. The impact of these realistic scenarios related to the current service network, related to the long-haul network design and selection of long-haul rail services offered, on operational transport costs can be evaluated to provide decision support in an intermodal transport planning environment. Finally, Section 6.3 concludes with managerial insights on the service network design.

\subsection{The Impact of Changes in the Service Network: Removing Long-Haul Services with Small Capacity}

Intermodal service operators decide which services they offer in order to minimise costs and satisfy expected service levels of current or future customers at a tactical level. The selected service network relates to characteristics of the transport requests, such as the customer locations. For example, the distance between customer locations and their closest terminal should not be too large.

Various reasons may exist for service network decisions on (not) opening certain long-haul service connections. Economies of scale can be obtained if less services are operated, resulting in a lower fixed cost for opening and maintaining these services, determined at the tactical decision level. However, if more terminals are operated, this may reduce vehicle routing costs at the operational level. Therefore, the impact of routing transport requests using a smaller number of long-haul services is evaluated. Within this context, long-haul service connections with a smaller capacity are excluded and its capacity is added to long-haul services which already own a larger share of the capacity. Two cases are studied in which each time one connection between two terminals is removed in comparison to the base scenario. Case $a$ omits the connection between terminal $1 C$ and $2 D$, which is the sole connection of $2 D$, while case $b$ excludes the connection between terminal $1 D$ and $2 C$, which is the only existing long-haul service from terminal $1 D$. The most realistic instance classes with a high capacity utilisation of $95 \%$ are used for these analyses. The capacity of the excluded long-haul service is added to the other remaining services to ensure that enough capacity is available for all requests. It is added to the remaining long-haul services in proportion to the current capacity share of each long-haul service.

Table 4 displays the average results for both alternative scenarios, $a$ and $b$, in comparison with the base scenario which consists of the current service network. Performance measures and the number of requests with departure and arrival in the current planning week are indicated.

Table 4. Detailed results for the removal of a long-haul connection relative to the base case, cases $a$ and $b$.

\begin{tabular}{llccc}
\hline & & Base & $\mathbf{a}$ & $\mathbf{b}$ \\
\hline \multirow{2}{*}{ Random } & TC_obj & $1,315,115.29$ & $1,316,977.03$ & $1,330,566.26$ \\
& TC_real & $144,115.29$ & $144,921.47$ & $148,366.26$ \\
& vrpCost & $108,755.37$ & $109,088.22$ & $111,905.62$ \\
& directTruckCost & $21,381.42$ & $21,959.91$ & $22,486.64$ \\
& Nbr requests (this week) & 257.10 & 253.57 & 255.78 \\
\hline \multirow{2}{*}{ Cluster } & TC_obj & $1,304,305.69$ & $1,314,235.72$ & $1,328,823.97$ \\
& TC_real & $137,305.69$ & $144,423.22$ & $147,712.86$ \\
& vrpCost & $103,223.35$ & $108,734.29$ & $111,374.22$ \\
& directTruckCost & $20,145.84$ & $21,832.68$ & $22,380.31$ \\
& Nbr requests (this week) & 256.30 & 253.52 & 255.67 \\
\hline
\end{tabular}

Generally, a slight increase in the number of load units with long-haul service arrival in the next week is observed in all cases, which partly explains the increase in the total cost objective function (TC_obj) in all cases. This also results in an increased two-way direct truck cost (directTruckCost). 
Due to a lower overall frequency of service obtained by omitting a long-haul service, a small number of requests cannot be feasibly assigned to a long-haul service arriving in the current planning week. Consequently, more load units arrive at their arrival terminal in the next planning cycle. Furthermore, the availability of fewer long-haul services results in fewer routing possibilities for each request, and higher terminal-to-customer distances, and as such, increases vehicle routing costs (vrpCost).

Operating a smaller number of services results in increased operational, variable transport costs. However, at the medium term, fixed costs for operating these services may be reduced. These costs are not included in the results. Case $b$ involves a higher increase in total costs, as the only connection to terminal $2 D$ (which is not centrally located) is excluded. In case $a$, two alternative connections are available to the terminals in Italy, while the only connection of terminal $1 D$ is removed. However, another terminal is closely located to terminal $1 D$, which explains the smaller increase in total costs. When comparing instances with respect to their customer locations, the increase in transport costs is clearly larger for clustered customer locations. For requests with random customer locations, the number of alternative, feasible long-haul services is often higher, resulting in more options and thus flexibility for routing trucks, and lower additional trucking costs.

To conclude, multiple reasons exist for (not) opening long-haul service connections between intermodal terminals. Operating to and from multiple terminals could results in reduced trucking costs at the operational level and more flexibility in the assignment of requests to long-haul services, and as such might increase customer service levels. If the locations of these terminals are spread across the service area, operations can be performed close to both customers and terminals. However, the more terminals are used for transport, the higher the expected fixed costs related to operating long-haul services between terminals. These costs are not included in the analyses. For a given demand volume, costs of a higher long-haul service frequency with a smaller capacity per departure-which may lead to a higher service level offered to customers-should be weighed against the costs of a low departure frequency and a higher capacity per departure. The latter is likely to reduce flexibility due to the fact that fewer long-haul services are available and may result in increased trucking costs.

\subsection{The Impact of Congestion around Terminals}

In practice, some terminals are much more congested compared to other terminals and service operators might tend to avoid such terminals. In this alternative case, the influence of congestion is analysed by means of a penalty time and cost for visiting the direct service area of a single busy terminal, in this case terminal $1 \mathrm{~A}$. The true costs of congestion may consist of truck driver wage costs, costs for not being in time-such as planning time and additional costs for finding alternative routing options-and customer dissatisfaction. Therefore, an additional cost of 30 Euro per hour of congestion is included for all trips to and from the congested terminal. As the amount of congestion is uncertain and unknown in advance, scenarios are included with the additional time varying from one hour to three hours, resulting in three congestion scenarios. The goal is to show how the integrated approach can be used on given input data to analyse the impact of congestion on planning decisions.

Experiments are conducted using a representative instance with respect to realistic input data. In real-life, customer locations are somewhere between random and clustered. Therefore, both classes are presented, such that practitioners can select the case which fits their customer base the most. To explore the full impact, experiments are performed using an instance with $85 \%$ utilisation to ensure that enough capacity is available at other long-haul connections. In this way, flexibility is allowed in order to show possible routing scenarios, which can be used as the basis for renegotiating transport contracts. These analyses may show at which connections capacity should be increased in order to avoid congested terminals.

Figure 11 displays the obtained results for an instance with random customer locations. Possibly impacted terminals with a direct connection to terminals $1 \mathrm{~A}$ are terminal $2 \mathrm{~A}$ and $2 \mathrm{C}$. The throughput share of each terminal within each service region is indicated, calculated based 
on the sum of inbound and outbound requests for each terminal. The base case represents the case without congestion.

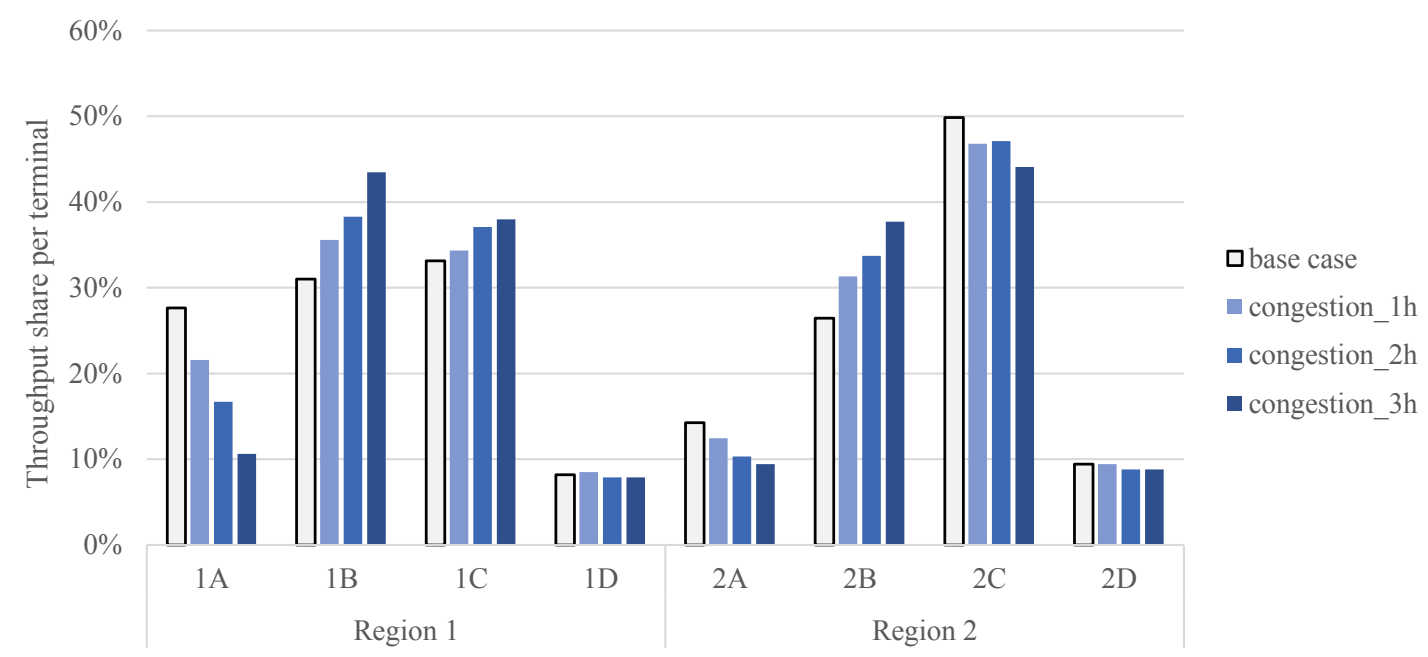

Figure 11. Difference in terminal throughput share due to congestion for random customer locations.

A structural decrease can be observed in the throughput of terminal $1 A$. The terminal usage decreases further when congestion time increases. Due to the random customer locations, an assignment to terminal $1 \mathrm{~A}$ might be required for some requests in order to be feasibly assigned and arrive in time at the customer destination location. Impacted terminals with a connection to terminal $1 A$ in service region 2 are terminals $2 A$ and $2 C$.

In the Benelux region, terminals $1 B$ and $1 C$ are alternative routing options. They are centrally located in the service region and present feasible alternatives for many requests. The throughput of terminal $1 B$ clearly increases the most as it is directly connected to one of the impacted terminals in the second region. In northern Italy, the relative throughput clearly increases in terminal $2 B$, which is also centrally located with multiple routing options. While terminal $2 C$ is also characterised by a centralized location, the throughput decreases due to its connection with the congested terminal. This effect outweighs the possible advantages of its central location. Furthermore, due to its direct link with the congested terminal, the share of terminal $2 A$ also slightly decreases. A steady throughput is observed for both terminals $1 D$ and $2 D$. They are not centrally located, they are less likely to present a feasible alternative for requests which were initially transported via terminal $1 A$ and both terminals each only have a long-haul service connection to a single other terminal.

Results for clustered customer locations show a similar pattern with respect to the impact of a single congested terminal. Detailed results are presented in Appendix B.

The above analysis provides insights in alternative routing decisions in order to avoid congested terminals.

\subsection{Managerial Insights}

Intermodal operators offer a service network through which transport requests are routed for the long-haul transport. Tactical decisions on the design of the service network are made in the long term using expectations about future demand, and transport contracts for operating long-haul services or purchased slots on long-haul services of other operators are fixed for multiple months. At the operational level, transport requests are routed through this given service network offered by the intermodal operator.

Usually, transport requests are assigned to the long-haul service with the smallest total terminal-to-customer distance. However, this might not always prove to be the best decision as not all information is accounted for. Vehicle routing costs are ignored, and initial assignments might not 
always be feasible, for example due to customer-depot distances or trucking and driving constraints. By taking an integrated perspective, infeasible long-haul routing decisions based on incomplete information can be avoided. By including information on feasible vehicle and long-haul routes and accounting for capacity restrictions on long-haul services and a heterogeneous truck and container fleet, the integrated approach aims at providing better-informed decisions in comparison with the traditional, sequential method. For example, when setting pricing decisions for customers based on more complete information, a more accurate price might be charged corresponding closer to real costs incurred. Furthermore, ideally, the service network should evolve in time in order to adapt to expected customer demand characteristics. The proposed integrated intermodal routing problem allows to evaluate the impact of variations in expected customer demand. Before accepting new customers, the integrated method could analyse the possible impact on transport costs and routing decisions. When renegotiating transport contracts on purchased slots, such evaluation can be used to propose adaptations to the service network accordingly.

\section{Discussion and Conclusions}

In this paper, potential savings and advantages of an integrated approach for intermodal routing are examined. The proposed integrated intermodal routing heuristic encourages a seamless integration of decisions on truck routing and long-haul intermodal service selection. A reduction of drayage costs and an improvement of the capacity utilisation stimulate the use of intermodal transport. While this study focuses on a two-region truck-rail network, the solution approach can deal with other transport modes and multiple regions.

It is demonstrated that cost savings can be obtained by the integrated approach in which decisions on truck routing and long-haul container routing are made simultaneously. Although cost savings are not obtained for instances with customer locations placed randomly in the service regions, more load units can be transported, indicating a better utilisation of the available long-haul capacity. Besides, for clustered instances, savings in trucking costs are obtained. Moreover, results indicate that a trade-off exists between increasing the capacity utilisation in the current planning week and reducing vehicle routing costs. Finally, computation times amount to a maximum of 1000 seconds, which is certainly acceptable for the design of the planning of an entire week.

Future work may focus on a dynamic version of the problem. Whereas the current approach serves the purpose of providing insights in the way the current service network design impacts total transport costs, a more operational approach considering planning on a daily basis could further support the day-to-day planning process. Furthermore, at the tactical level, the service network and its characteristics are variable. To cope with occasional capacity shortages, overflow can be assigned to one-time purchased slots at an additional cost. By optimally managing own services and services offered by other operators, total costs of operating the transport system could be further reduced.

Author Contributions: H.H. wrote the original draft of the paper and developed the LNS heuristic to solve the integrated intermodal routing problem; Y.M. and K.B. participated in setting up the design of the heuristic algorithm; A.C. and K.B. supervised the study, provided comments and major edits.

Funding: Yves Molenbruch is a postdoctoral researcher funded by the Research Foundation Flanders (FWO), grant number $1202719 \mathrm{~N}$.

Acknowledgments: This work is supported by the Strategic Basic Research project Data-driven logistics (S007318N), funded by the Research Foundation Flanders (FWO). The computational resources and services used in this work were provided by the VSC (Flemish Supercomputer Center), funded by the Research Foundation-Flanders (FWO) and the Flemish Government-department EWI.

Conflicts of Interest: The authors declare no conflict of interest. 


\section{Appendix A. Parameter Tuning}

\section{Appendix A.1. Tuning the Parameter Values}

To determine a parameter setting, tests are executed using various parameter values for both the sequential and the integrated approach. Table A1 shows the tested parameter values of the large neighbourhood search heuristic.

Table A1. LNS algorithm parameters for tuning.

\begin{tabular}{cc}
\hline Input Parameter & Tested Values \\
\hline Number of iterations & {$[0 ; 3000]$} \\
Removal of requests & $5 \% ; 10 \% ; 15 \%$ \\
Acceptance threshold of new solutions $d e v$ & $0 \% ; 1 \% ; 3 \% ; 5 \%$ \\
\hline
\end{tabular}

Experimental results for the parameter tuning phase with respect to the solution quality are summarised in Figure A1. Computation times are visualised for both approaches in Figure A2 after 3000 iterations.

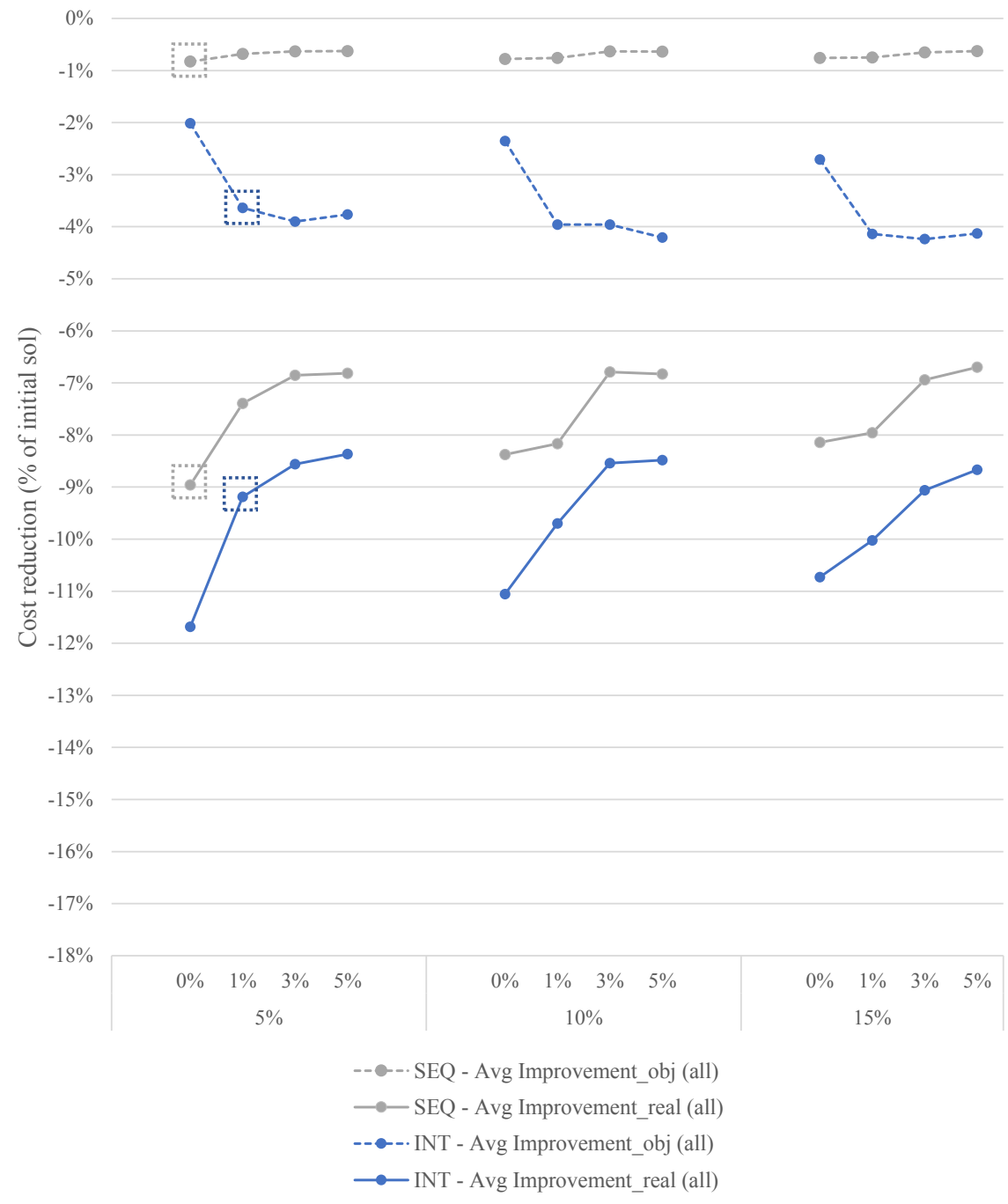

Figure A1. LNS parameter tuning: average solution quality (reduction in total costs compared to initial solution) for the sequential (SEQ) and integrated (INT) approach after 3000 iterations; Improvement_obj = reduction in total costs including high value for load units with long-haul service arrival in next planning cycle; Improvement_real= reduction in real total costs. 
For each combination of parameter values, Figure A1 displays the percentage reduction in the objective function value obtained after 3000 iterations in comparison with the initial solution found by the constructive heuristic on the vertical axis, averaged over all instances. The lower on the vertical axis, the larger the cost reduction, and the higher the solution quality. The initial solution is identical in both approaches for a single instance. On the horizontal axis, the percentage of requests or tasks that is removed from the solution is indicated in the bottom. Above each removal percentage, variations in the allowed deviation for accepting new moves are reported. Results of the sequential and integrated approach are shown in grey and blue respectively. Dotted lines indicate the solution quality of the objective function, while real total costs are illustrated using solid lines. The difference between both performance measures relates to the artificial, high value for load units assigned to long-haul services with arrival in the next planning cycle. This high value is included in the objective function, but excluded from the real total cost.

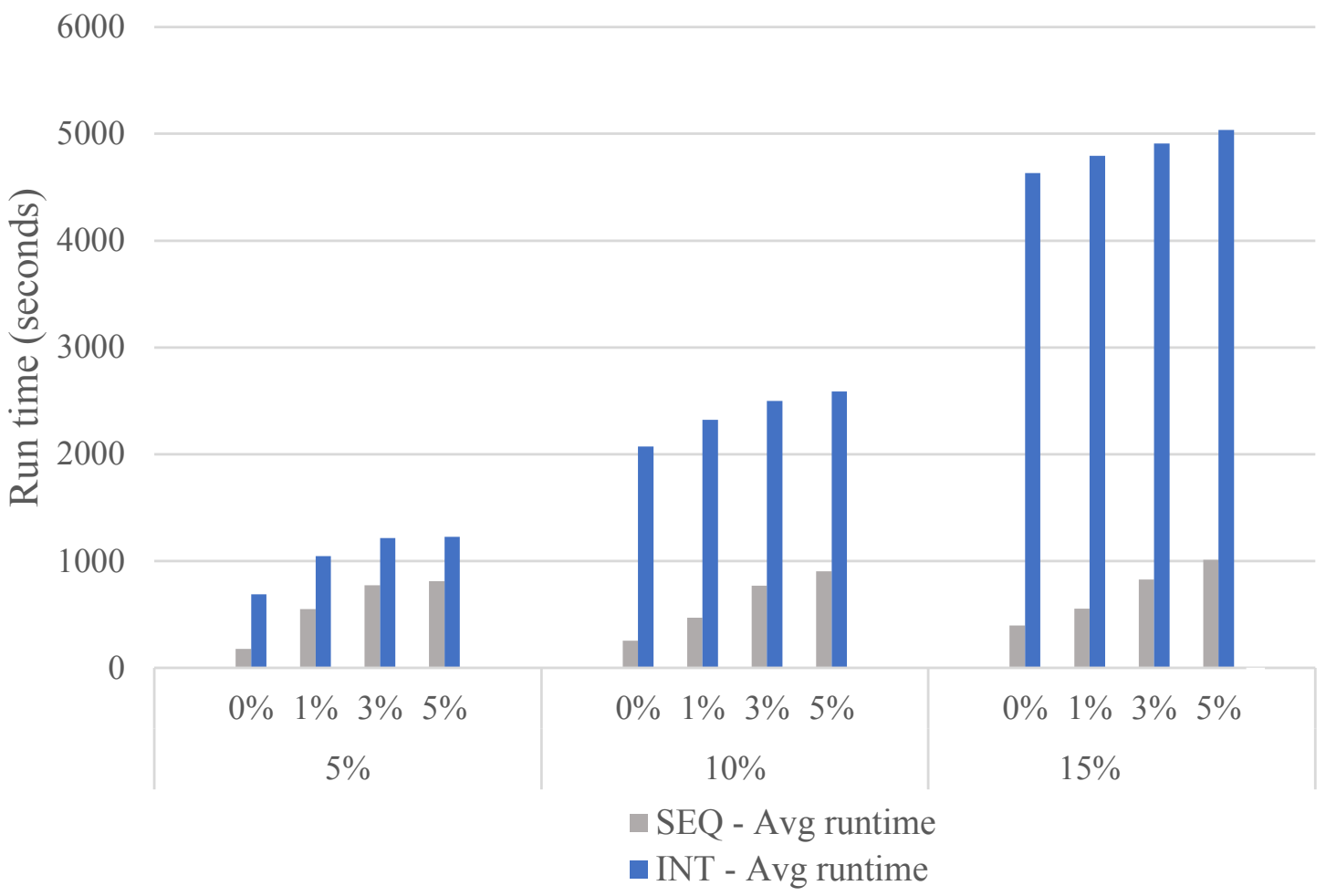

Figure A2. LNS parameter tuning: average computation time for the sequential (SEQ) and integrated (INT) approach.

Generally, it can be observed that, when the allowed deviation from the current best solution is too small, changes in the long-haul service assignments are not possible, and reductions in the objective value are small. On the other hand, if the allowed deviation is too big, this has a negative impact on the costs of truck routes, and thus on the real total costs.

For each parameter setting, the integrated setting (blue lines) shows a larger percentage reduction in the objective function (dotted lines) as well as total costs (solid lines) in comparison with the sequential setting (grey lines). This is partly due to the fact that more load units can be assigned to this week's long-haul services. For the same parameter setting, total costs for drayage operations are always smaller in an integrated approach, and more drayage tasks are performed in the current planning week.

Tuning results for the sequential approach show that small improvements in the objective value are observed compared to the initial solution found by the constructive heuristic (indicated by the grey dotted lines) for all combinations of parameter settings. This demonstrates that the quality of the initial solution seems to be rather high. The real total costs (grey solid lines) consistently increase 
as the allowed deviation for accepting new solutions increases. This can be observed for each value of the removal percentage. A similar pattern is observed for the objective function (grey dotted lines). A slight decrease in the performance of the sequential heuristic is observed if the removal percentage is increased above $5 \%$. This may be due to the fact that a small removal percentage already relates to a high absolute number of drayage tasks. Based on these observations, it can be concluded that total costs rise while the number of tasks to plan does not change when either the acceptance threshold or the removal percentage increases. Besides, computation times increase when removing more drayage tasks. As the graph demonstrates a good overall performance when accepting only improving solutions, and real total costs are minimised at a $5 \%$ removal rate, a $0 \%$-acceptance threshold in combination with a removal of $5 \%$ of the drayage tasks leads on average to a minimum-cost solution for the sequential approach.

For the integrated version of the heuristic, results show larger differences in the objective and total cost values for varying parameter settings. For the objective function, it is clear that accepting improving solutions only (i.e., $0 \%$ deviation) results in the worst objective value. When looking at the overall average real total cost (given by the blue solid lines), it can be concluded that a $1 \%$ deviation is the best choice. Other values for the allowed deviation when accepting new solutions ( $3 \%$ and $5 \%)$ result in the same objective value, at a higher real total cost. With respect to the different removal percentages, a very slight reduction in the objective value is observed when increasing the removal percentage. As results are quite similar and the computation time increases substantially when increasing the removal percentage, a $5 \%$ removal is selected.

\section{Appendix A.2. The Stopping Criterion}

Next, the stopping criterion is analysed. Figure A3 shows the relationship between the solution quality and the number of executed iterations. The solution quality is measured by means of the average percentage improvement (i.e., reduction) in the objective value, and thus the artificial, high values for load units assigned to long-haul services with arrival in the next planning cycle are included. It is an average over all replications and all instances within a single instance class.

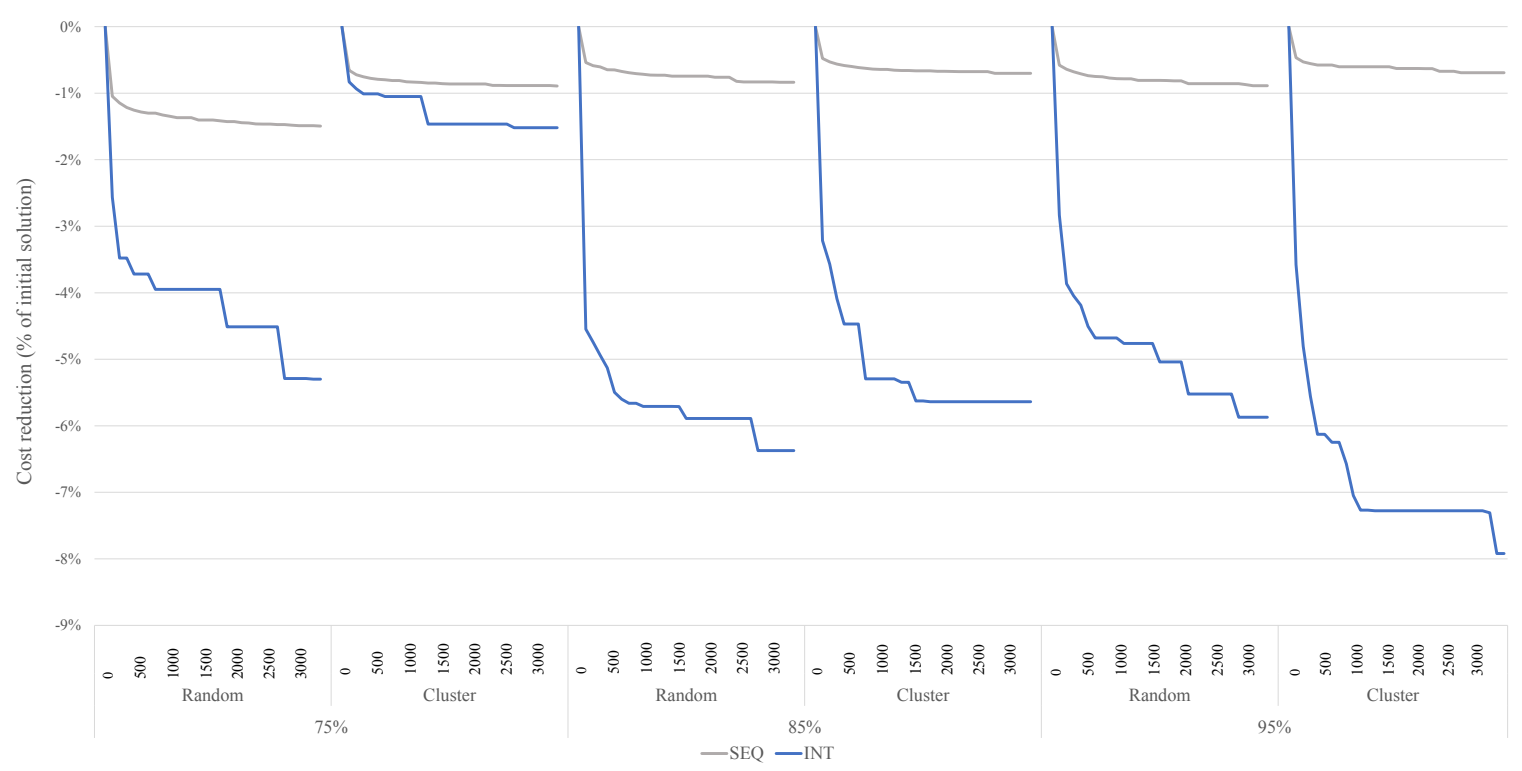

Figure A3. LNS parameter tuning: evolution of the solution quality with the number of iterations.

It can be observed that the largest improvements are obtained for realistic instances with a demand at $95 \%$ of the available capacity, and thus the integrated approach is most advantages for these realistic instances. In the integrated approach, the solution quality increases consistently and flattens out around 3000 iterations. The solution quality of the sequential heuristic levels out much earlier. However, 
in order to maintain a fair comparison, 3000 iterations are used for both approaches. Within the aim of this study, computation time is not the most critical criterion in order to demonstrate the difference between two approaches. If the solution approach is to be used in practice and computation time is an issue, one could easily adapt the stopping criterion to a maximum allowed computation time.

\section{Appendix B. Results for the Congestion Scenario with Clustered Customer Locations}

Figure A4 visualizes the throughput changes due to congestion for clustered customer locations.

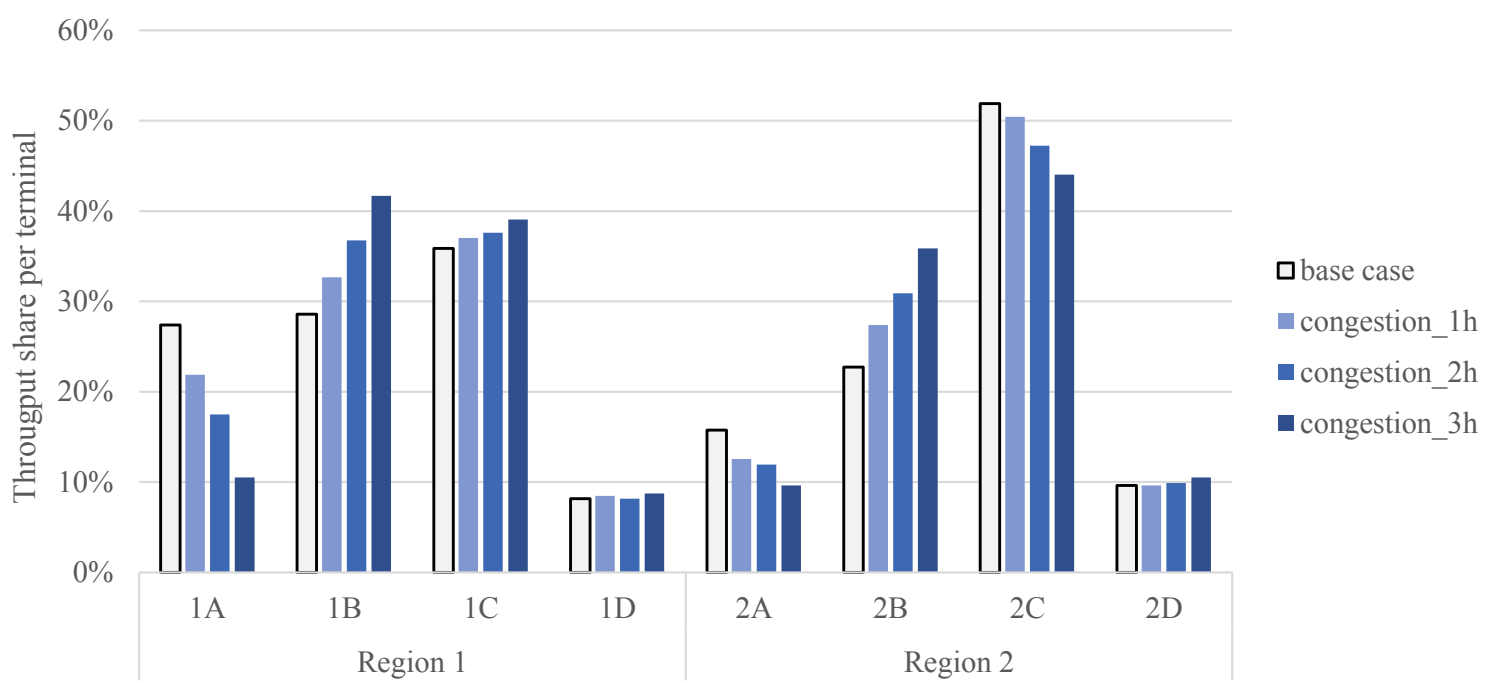

Figure A4. Difference in terminal throughput share due to congestion for clustered customer locations.

Results for clustered customer locations show a similar pattern as random customer locations with respect to the impact of a single congested terminal. In the Benelux, the relative increase in the usage of terminal $1 B$ is larger. Due to the clustered request customer locations and the centralized location, this terminal might provide an interesting, feasible alternative for more requests impacted due to congestion. For terminal $1 C$, the impact is smaller, as in the base case, its share is already relatively high. In the second service region, the decrease in the importance of terminal $2 C$ is even larger.

\section{References}

1. Eurostat. Modal Split of Freight Transport, 2016. Available online: https:/ / ec.europa.eu/eurostat/web/ products-datasets / product?code=t2020_rk320 (accessed on 14 March 2019).

2. European Commission. MEMO/11/197: Transport 2050: The Major Challenges, the Key Measures, 2011. Available online: http://europa.eu/rapid/press-release_MEMO-11-197_en.htm (accessed on 14 March 2019).

3. ITF. ITF Transport Outlook 2015; ITF Transport Outlook; OECD Publishing: Paris, France, 2015.

4. European Commission. White Paper: A Sustainable Future for Transport-Towards an Integrated, Technology-Led and User Friendly System, 2009. Available online: https:/ / ec.europa.eu/transport/themes/ strategies/consultations/2009_09_30_future_of_transport_en (accessed on 14 March 2019).

5. Alliance for Logistics Innovation through Collaboration in Europe. Corridors, Hubs and Synchromodality Research and Innovation Roadmap, 2014. Available online: https://www.etp-logistics.eu/wp-content/ uploads/2015/08/W26mayo-kopie.pdf (accessed on 14 March 2019).

6. Montreuil, B. Toward a Physical Internet: Meeting the global logistics sustainability grand challenge. Logist. Res. 2011, 3, 71-87. [CrossRef]

7. Ambra, T.; Caris, A.; Macharis, C. Towards freight transport system unification: Reviewing and combining the advancements in the physical internet and synchromodal transport research. Int. J. Prod. Res. 2018, 1-18. [CrossRef] 
8. Caris, A.; Macharis, C.; Janssens, G.K. Planning Problems in Intermodal Freight Transport: Accomplishments and Prospects. Transp. Plan. Technol. 2008, 31, 277-302. [CrossRef]

9. Caris, A.; Macharis, C.; Janssens, G.K. Decision support in intermodal transport: A new research agenda. Comput. Ind. 2013, 64, 105-112. [CrossRef]

10. Crainic, T.G.; Kim, K.H. Chapter 8 Intermodal Transportation. In Handbooks in Operations Research and Management Science; Elsevier: Amsterdam, The Netherlands, 2007; Volume 14, pp. 467-537.

11. Li, L.; Negenborn, R.R.; De Schutter, B. Intermodal freight transport planning-A receding horizon control approach. Transp. Res. Part C: Emerg. Technol. 2015, 60, 77-95. [CrossRef]

12. SteadieSeifi, M.; Dellaert, N.; Nuijten, W.; Van Woensel, T.; Raoufi, R. Multimodal freight transportation planning: A literature review. Eur. J. Oper. Res. 2014, 233, 1-15. [CrossRef]

13. Heggen, H.; Braekers, K.; Caris, A. A multi-objective approach for intermodal train load planning. OR Spectr. 2018, 40, 341-366. [CrossRef]

14. van Riessen, B.; Negenborn, R.R.; Dekker, R. Real-time container transport planning with decision trees based on offline obtained optimal solutions. Decis. Support Syst. 2016, 89, 1-16. [CrossRef]

15. Zhang, M.; Pel, A. Synchromodal hinterland freight transport: Model study for the port of Rotterdam. J. Transp. Geogr. 2016, 52, 1-10. [CrossRef]

16. Grasman, S.E. Dynamic approach to strategic and operational multimodal routing decisions. Int. J. Logist. Syst. Manag. 2006, 2, 96-106. [CrossRef]

17. Bock, S. Real-time control of freight forwarder transportation networks by integrating multimodal transport chains. Eur. J. Oper. Res. 2010, 200, 733-746. [CrossRef]

18. Di Febbraro, A.; Sacco, N.; Saeednia, M. An agent-based framework for cooperative planning of intermodal freight transport chains. Transp. Res. Part C Emerg. Technol. 2016, 64, 72-85. [CrossRef]

19. Erera, A.L.; Morales, J.C.; Savelsbergh, M. Global intermodal tank container management for the chemical industry. Transp. Res. Part E Logist. Transp. Rev. 2005, 41, 551-566. [CrossRef]

20. Assadipour, G.; Ke, G.Y.; Verma, M. Planning and managing intermodal transportation of hazardous materials with capacity selection and congestion. Transp. Res. Part E Logist. Transp. Rev. 2015, 76, 45-57. [CrossRef]

21. Jiang, X.; Bai, R.; Atkin, J.; Kendall, G. A scheme for determining vehicle routes based on Arc-based service network design. INFOR Inf. Syst. Oper. Res. 2017, 55, 16-37. [CrossRef]

22. Verma, M.; Verter, V.; Zufferey, N. A bi-objective model for planning and managing rail-truck intermodal transportation of hazardous materials. Transp. Res. Part E Logist. Transp. Rev. 2012, 48, 132-149. [CrossRef]

23. Moccia, L.; Cordeau, J.F.; Laporte, G.; Ropke, S.; Valentini, M.P. Modeling and solving a multimodal transportation problem with flexible-time and scheduled services. Networks 2011, 57, 53-68. [CrossRef]

24. Chang, T.S. Best routes selection in international intermodal networks. Comput. Oper. Res. 2008, 35, $2877-2891$. [CrossRef]

25. Caramia, M.; Guerriero, F. A heuristic approach to long-haul freight transportation with multiple objective functions. Omega 2009, 37, 600-614. [CrossRef]

26. van Riessen, B.; Negenborn, R.R.; Dekker, R.; Lodewijks, G. Service network design for an intermodal container network with flexible transit times and the possibility of using subcontracted transport. Int. J. Shipp. Transp. Logist. 2015, 7, 457-477. [CrossRef]

27. Rudi, A.; Fröhling, M.; Zimmer, K.; Schultmann, F. Freight transportation planning considering carbon emissions and in-transit holding costs: A capacitated multi-commodity network flow model. Euro J. Transp. Logist. 2016, 5, 123-160. [CrossRef]

28. Caris, A.; Janssens, G. A local search heuristic for the pre- and end-haulage of intermodal container terminals. Comput. Oper. Res. 2009, 36, 2763-2772. [CrossRef]

29. Toth, P.; Vigo, D. (Eds.) Vehicle Routing: Problems, Methods, and Applications, 2nd ed.; Number 18 in MOS-SIAM Series on Optimization; SIAM [u.a.]: Philadelphia, PA, USA, 2014.

30. Braekers, K.; Ramaekers, K.; Van Nieuwenhuyse, I. The vehicle routing problem: State of the art classification and review. Comput. Ind. Eng. 2016, 99, 300-313. [CrossRef]

31. Dotoli, M.; Epicoco, N. A technique for the optimal management of containers' drayage at intermodal terminals. In Proceedings of the 2016 IEEE International Conference on Systems, Man, and Cybernetics (SMC), Budapest, Hungary, 9-12 October 2016; pp. 566-571. 
32. Pérez Rivera, A.E.; Mes, M.R.K. Scheduling Drayage Operations in Synchromodal Transport. In Computational Logistics; Bektaş, T., Coniglio, S., Martinez-Sykora, A., Voß, S., Eds.; Springer International Publishing: Cham, Switzerland, 2017; Volume 10572, pp. 404-419.

33. Zhang, R.; Yun, W.Y.; Moon, I. A reactive tabu search algorithm for the multi-depot container truck transportation problem. Transp. Res. Part E Logist. Transp. Rev. 2009, 45, 904-914. [CrossRef]

34. Zhang, R.; Yun, W.Y.; Kopfer, H. Heuristic-based truck scheduling for inland container transportation. OR Spectr. 2010, 32, 787-808. [CrossRef]

35. Nossack, J.; Pesch, E. A truck scheduling problem arising in intermodal container transportation. Eur. J. Oper. Res. 2013, 230, 666-680. [CrossRef]

36. Sterzik, S.; Kopfer, H. A Tabu Search Heuristic for the Inland Container Transportation Problem. Comput. Oper. Res. 2013, 40, 953-962. [CrossRef]

37. Reinhardt, L.B.; Pisinger, D.; Spoorendonk, S.; Sigurd, M.M. Optimization of the drayage problem using exact methods. INFOR: Inf. Syst. Oper. Res. 2016, 54, 33-51. [CrossRef]

38. Shiri, S.; Huynh, N. Optimization of drayage operations with time-window constraints. Int. J. Prod. Econ. 2016, 176, 7-20. [CrossRef]

39. Francis, P.; Zhang, G.; Smilowitz, K. Improved modeling and solution methods for the multi-resource routing problem. Eur. J. Oper. Res. 2007, 180, 1045-1059. [CrossRef]

40. Braekers, K.; Caris, A.; Janssens, G.K. Integrated planning of loaded and empty container movements. OR Spectr. 2013, 35, 457-478. [CrossRef]

41. Posada, M.; Andersson, H.; Häll, C.H. The integrated dial-a-ride problem with timetabled fixed route service. Public Transp. 2017, 9, 217-241. [CrossRef]

42. Medina, J.; Hewitt, M.; Lehuédé, F.; Péton, O. Integrating long-haul and local transportation planning: The Service Network Design and Routing Problem. EURO J. Transp. Logist. 2018. [CrossRef]

43. Dragomir, A.G.; Nicola, D.; Soriano, A.; Gansterer, M. Multidepot pickup and delivery problems in multiple regions: A typology and integrated model. Int. Trans. Oper. Res. 2018, 25, 569-597. [CrossRef]

44. Soriano, A.; Gansterer, M.; Hartl, R.F. The two-region multi-depot pickup and delivery problem. OR Spectr. 2018. [CrossRef]

45. Wolfinger, D.; Tricoire, F.; Doerner, K.F. A matheuristic for a multimodal long haul routing problem. EURO J. Transp. Logist. 2018. [CrossRef]

46. Shaw, P. Using Constraint Programming and Local Search Methods to Solve Vehicle Routing Problems. In Principles and Practice of Constraint Programming—CP98; Goos, G., Hartmanis, J., van Leeuwen, J., Maher, M., Puget, J.F., Eds.; Springer: Berlin/Heidelberg, Germany, 1998; Volume 1520, pp. 417-431.

47. Ropke, S.; Pisinger, D. An Adaptive Large Neighborhood Search Heuristic for the Pickup and Delivery Problem with Time Windows. Transp. Sci. 2006, 40, 455-472. [CrossRef]

48. Savelsbergh, M.W.P. The Vehicle Routing Problem with Time Windows: Minimizing Route Duration. ORSA J. Comput. 1992, 4, 146-154. [CrossRef]

49. Vidal, T.; Crainic, T.G.; Gendreau, M.; Prins, C. Timing problems and algorithms: Time decisions for sequences of activities. Networks 2015, 65, 102-128. [CrossRef]

50. Cordeau, J.F.; Gendreau, M.; Laporte, G. A tabu search heuristic for periodic and multi-depot vehicle routing problems. Networks 1997, 30, 105-119. [CrossRef]

(C) 2019 by the authors. Licensee MDPI, Basel, Switzerland. This article is an open access article distributed under the terms and conditions of the Creative Commons Attribution (CC BY) license (http://creativecommons.org/licenses/by/4.0/). 